\title{
37. STAR CLUSTERS AND ASSOCIATIONS
}

\section{(AMAS STELLAIRES ET ASSOCIATIONS)}

President: G. Larsson-Leander.

ViCE-PRESIDENT: I. R. King.

Organizing Commitree: K. A. Barkhatova, S. C. B. Gascoigne, M. Golay, L. Rosino, J. Ruprecht, S. van den Bergh, H. van Schewick (†), M. F. Walker.

\section{INTRODUCTION}

The outline of the present report of Commission 37 is somewhat different from previous ones. The traditional tabulations of published papers and current programmes are considered most useful, and efforts have been made to make them as complete as possible. Drs. Barkhatova and Kuz'mina prepared a first version of Table 1, which was edited and considerably enlarged by me. Dr King, Commission Vice-President, wrote Section 6, and Drs. van den Bergh and Walker, of the Organizing Committee, wrote 4D and 7A, respectively. These contributions appear essentially unchanged in the report, except for addition of occasional material and editing, to obtain homogeneity. Drs. Barkhatova and Kuz'mina sent a comprehensive review on cluster and association work in the U.S.S.R. Following a request, Dr R. E. White kindly prepared a summary on globular-cluster work. It arrived late, but gave some additional data for Table 3. Many Commission members and other colleagues furnished me with more or less extensive progress reports. For all this help I am most grateful. I regret that due to the limited space, most of the reports had to be shortened drastically.

To minimize the space needed for references, published papers are, whenever possible, identified by the entry numbers in Astronomy and Astrophysics Abstracts.

Commission 37 suffered a great loss by the untimely death of Dr H. van Schewick, on September 6,1972 . Dr van Schewick was a Member of the present Organizing Committee, and he was as the time of his death engaged in extensive studies of proper motions of open clusters. It is also with deep regret than we record the death, on October 20,1972, of Dr Harlow Shapley, pioneer in the study of globular clusters and the first President of Commission 37, and the death of Dr G. A. Alter on October 30, 1972.

\section{SYMPOSIA AND COLLOQUIA}

Since the 1970 report several symposia and other conferences have been devoted to topics of interest to the Commission. The following ones are especially to be mentioned:

(1) IAU Colloquium No. 10, 'Gravitational $N$-body Problem', August 1970, Cambridge, England. The proceedings (ed. by M. Lecar) were published by D. Reidel Publ. Co., Dordrecht, 1972.

(2) Conference on 'The Evolution of Population II Stars', December 1970, State University of New York. The proceedings (ed. by A. G. D. Philip) appeared as Dudley Obs. Report, No. 4, 1972.

(3) IAU Colloquium No. 21, 'Variable Stars in Globular Clusters and Related Systems', August 1972, Richmond Hill, Canada.

(4) IAU Symposium No. 54, 'Problems of Absolute Magnitudes and Temperatures of Stars', September 1972, Geneva, Switzerland.

(5) IAU Colloquium No. 17, 'Ages des Étoiles', September 1972, Meudon, France.

The Commission was a co-sponsor of IAU Symposium No. 54 and took an active part in the realization of Colloquium No. 17. The Commission is co-sponsoring the forthcoming Symposium No. 62, 'The Stability of the Solar System and of Small Stellar Systems', to be held September 1973 in Warsaw, Poland. 


\section{Catalogues and atlases}

The second, considerably enlarged edition of the Catalogue of Star Clusters and Associations (ed. by G. Alter, B. Balazs, and J. Ruprecht) was printed in 1970 by the Publishing House of the Hungarian Academy of Sciences, Budapest. Supplements will be issued at intervals. To facilitate compilation of supplementary material preprints or at least abstracts of relevant papers should be sent to the Editors (preferably to Dr Balazs).

Another very valuable source of information is the atlas of colour-magnitude diagrams for open clusters, published by Hagen (04.153.050). The Atlas contains 189 cards, giving c-m diagrams for the same number of clusters, all observed on the $U B V$ or $U_{\mathrm{c}} B V$ system by photoelectric or photoelectric plus photographic techniques.

Becker and Fenkart (06.153.015) published a new catalogue of open clusters observed in three colours $\left(U B V, U_{\mathrm{c}} B V\right.$, or $\left.R G U\right)$, giving distances and other cluster parameters. It contains 216 entries.

As mentioned in the 1970 report, White (03.154.014) compiled a bibliography of $c-m$ diagrams for globular clusters. Complete references are given up to August 1969, and certain c-m diagrams are reproduced.

Kukarkin (Moscow) is preparing a General Catalogue of Globular Clusters, which will give integrated magnitudes and colours, spectral types, diameters, etc., all reduced to uniform systems. The probable time of publication is 1974 .

It is expected (07.152.012) that the massive work of collecting and collating data from various catalogues and other sources, which is now in progress at the Centre de Données Stellaires in Strasbourg, will be of great service and considerably forward research on clusters and associations. As a beginning, Mermilliod (07.041.037) is preparing transit tables for several clusters, giving the correspondence in stellar enumeration adopted by various investigators. Up to October 1972 such tables were complete for 31 open clusters. Mermilliod has also made a catalogue of HD(E) and DM identifications for stars in 119 open clusters.

\section{NEW OBSERVATIONAL DATA AND PROGRAMMES}

This section contains the traditional tables, giving separately for each object information on current programmes and published observational papers. Omitted from the tabulations are still uncompleted programmes mentioned already in the 1970 report, as well as papers and programmes dealing with individual stars studied for their own interest. Most work on variables falls under this category.

In presenting the material the nomenclature and order of the Catalogue of Star Clusters and Associations is followed. This means that the Scorpius-Centaurus Association is regarded as a moving cluster and is included in 4B, together with other moving clusters and stellar groups. The stellar rings are included in $4 \mathrm{~A}$.

\section{A. Galactic associations}

Published papers and observing programmes on individual associations in our Galaxy are listed in Table 1. For most programmes both the home institute and the observing site are given.

Among the investigations listed in Table 1 those of Sco OB 1 by Laval $(07.152 .007 ; 9)$ should be especially mentioned. For the stellar study photometric and astrometric data down to $V=11$ were completed by spectrographic observations to the same magnitude, giving spectral classes and radial velocities. The characteristics of the ionized hydrogen were determined by interferometric $\mathrm{H} \alpha$-observations and by data obtained in radio astronomy.

In their study of Vul OB 1 and Vul OB 4, mentioned in the 1970 report, Thé and van Paradijs (05.152.011) found that eight out of nine stars in the region with known luminosity classes I and II are members of Vul OB 1 . The existence of Vul OB 4 seems doubtful.

Drilling (8) identified 99 OB stars in a survey of 20 sq. deg. in Norma. Most of these stars are 
Table 1. Associations

\begin{tabular}{|c|c|c|c|}
\hline Name & Observer and data obtained & Name & Observer and data obtained \\
\hline Cyg OB 2 & Jarecke $(07.152 .006) I \mathrm{pg}, I<14.5$ & & Vitrichenko (4) peculiarities faint \\
\hline \multirow{4}{*}{ Cep OB 3} & $\begin{array}{l}\text { Coyne et al. }(01.152 .001) \text { survey, pg, } \\
\text { s.t. } \\
\text { Lesh (02.152.002) kinematics }\end{array}$ & Ori OB 1a & $\begin{array}{l}\text { stars } \\
\text { Ciatti, Bernacca }(05.152 .002) \mathrm{He} \\
\text { weak-line st. }\end{array}$ \\
\hline & Weaver $(04.152 .003)$ obj.-pr. survey & Ori OB $1 b$ & Ciatti, Bernacca (5) He weak-line st. \\
\hline & $\begin{array}{l}\text { Crawford, Barnes (04.152.004) uvby } \beta \\
\text { Garmany (1) p.m., kinematics }\end{array}$ & Ori OB 1c & $\begin{array}{l}\text { Smith (6) uvby, spectra, Am st. } \\
\text { Smith (7) circumstellar gas em. }\end{array}$ \\
\hline & $\begin{array}{l}\text { Garrison }(04.152 .006) \mathrm{MK}, \mathrm{HR} \text {, } \\
R \text { value }\end{array}$ & Mon OB 1 & $\begin{array}{l}\text { Heiser (Dyer, KPNO) } U B V \text { pe, } \\
V<10.0\end{array}$ \\
\hline Cep OB 4 & $\begin{array}{l}\text { Churchwell, Felli }(03.152 .010) \text { comp. } \\
\text { radio obs. NGC } 7822\end{array}$ & Mon OB 2 & $\begin{array}{l}\text { Heiser (Dyer, KPNO) } U B V \text { pe, } \\
V<10 \cdot 0\end{array}$ \\
\hline Cas III & Bartaya, Kharadze $(03.152 .003)$ s.t. & CMa OB 1 & Claria (Cordóbá, CTIO) $U B V$ pe \\
\hline Cas OB 4 & Bartaya, Kharadze (03.152.003) s.t. & & Olmedo $(05.152 .013)$ \\
\hline Cas $\mathrm{OB} 7$ & Bartaya, Kharadze $(03.152 .003)$ s.t. & Pup OB 2 & Grubissich $(01.152 .003) R$ value \\
\hline Cas OB 8 & $\begin{array}{l}\text { Humphreys (Steward, KPNO) } U B V \\
\text { pe, MK }\end{array}$ & & $\begin{array}{l}\text { Havlen }(07.155 .024) U B V \beta \text { pe, } \\
\text { OB st. }\end{array}$ \\
\hline \multirow[t]{2}{*}{ Per OB 1} & $\begin{array}{l}\text { Gahm, Arkling (05.153.003) r.v., } \\
\text { red sg. }\end{array}$ & Pup OB 1 & $\begin{array}{l}\text { Havlen }(07.155 .024) U B V \beta \text { pe, } \\
\text { OB st. }\end{array}$ \\
\hline & $\begin{array}{l}\text { Mirzoyan, Mnatsakanian (04.153.033) } \\
\text { space vel. }\end{array}$ & Car OB 2 & $\begin{array}{l}\text { Seggewiss }(03.152 .014) U B V \text { pe }+ \text { pg, } \\
V<14.2\end{array}$ \\
\hline \multirow{5}{*}{$\begin{array}{l}\text { Cam OB } 1 \\
\text { Per OB } 2\end{array}$} & Bartaya, Kharadze (03.152.004) s.t. & Nor OB 1 & Drilling (8) $U B V \beta$ pe \\
\hline & Lesh $(02.152 .002)$ kinematics & Sco OB 1 & J. V. Barnes (KPNO, CITO) spect \\
\hline & Lynds (02.152.006) interstellar matter & & Crawford et al. (06.153.025) uvby \\
\hline & Rydgren (06.113.043) uvby $\beta$ & & $3.152 .001)$ r.v. \\
\hline & Sancisi (03.152.012) 21-cm obs. & & Laval (07.152.007) H II regions \\
\hline \multirow[t]{6}{*}{ Ori OB 1} & Heiser (02.152.008) $\mathrm{H} \beta$ phot. & & Laval (9) stellar kinematics \\
\hline & $\begin{array}{l}\text { Hesser, Henry (06.113.053) K-line } \\
\text { phot. }\end{array}$ & & $\begin{array}{l}\text { MacConnell, Perry (01.152.006) H } \alpha \\
\text { em. stars }\end{array}$ \\
\hline & Knacke et al. (2) IR studies & & Schild et al. (01.152.004) UBV pe, \\
\hline & Penston (3) 9-col., pe, $V<12$ & & MK, HR \\
\hline & $\begin{array}{l}\text { Sincheskul (06.152.009) catalogue: } \\
\text { mag., C.I., s.t. }\end{array}$ & & $\begin{array}{l}\text { Schild et al. (05.152.005) UBV pe, } \\
\text { MK, HK flux }\end{array}$ \\
\hline & $\begin{array}{l}\text { Strom, Strom (N.Y. State, KPNO) } \\
\text { image-tube sp. }\end{array}$ & $\begin{array}{l}\text { And T } 1 \\
\text { Oph T } 1\end{array}$ & $\begin{array}{l}\text { Shiukashvili }(03.152 .005) \text { s.t. } \\
\text { Shiukashvili }(03.152 .005) \text { s.t. }\end{array}$ \\
\hline
\end{tabular}

Abbreviations: $\mathrm{HR}=\mathrm{HR}$ diagram, $\mathrm{MK}=\mathrm{MK}$ classification, p.m. $=$ proper motions, r.v. $=$ radial velocities, sg. $=$ super-giants, sp. $=$ spectra, st. $=$ stars, s.t. $=$ low-dispersion spectral types.

associated with two visual concentrations of $\mathrm{OB}$ stars previously noted by Lyngå. One of the concentrations is Nor $\mathrm{OB} 1$, the other, at $l=332: 1, b=-1 \% 1$, is caused by two or more features seen in projection.

Bok and his collaborators at Steward Observatory discovered a small OB association near $l=275^{\circ}, b=-2^{\circ}$, containing at least six stars in the range $V=11 \cdot 7$ to $14 \cdot 8$. The minimum distance of the association is $6 \mathrm{kpc}$, and it is affected by $3 \mathrm{mag}$. of visual absorption. Graham (06.113.049) found that eleven faint $\mathrm{OB}$ stars in a field of $l=218^{\circ}, b=-0.5$, in Monoceros form two groups at different distances.

Several observational studies were made of the so-called stellar rings. Isserstedt (04.114.017) produced photometric evidence that ring No. 274, around P Cyg, represents a group of coeval stars, and the same claim was made for ring No. 373 by Isserstedt and Schmidt-Kaler (04.152.002). Additional photometry by Kolesnik $(07.152 .001)$ confirmed that some of the stars in No. 274 may 
form a physical group, while spectral classifications by Haug and Kohoutek (05.152.001), and by Preston (07.152.002), deny this possibility for ring No. 373 .

More complete information is available for the three nearby rings in Aquila, Hydra, and Orion. Isserstedt (02.152.004) concluded from photoelectric photometry that the Aquila ring represents a physical group of young stars. Radial velocities and spectroscopic distance-moduli, obtained by Crampton and Byl (05.152.012), and by Webster (10), show, however, that the aggregate is probably a chance superposition of stars at different distances. The reality of the Hydra ring as a physical entity was denied by Schild and Cowley $(06.152 .001)$ by means of new MK spectral classes, but they support the idea that the stars of the Orion ring belong to a physical group. Crampton and Byl $(05.152 .012)$ found the Orion-ring stars indistinguishable from field stars in the region. Taken as a whole, the observational evidence is not favourable for the concept of stellar rings of uniform size. Meurers (07.152.010) has also shown that ring features, like the observed ones, are easily produced by chance in two-dimensional point distributions.

\section{B. Galactic open clusters}

In Table 2, listing papers and programmes on galactic open clusters, and arranged according to the Catalogue of Star Clusters and Associations, the moving clusters and stellar groups are found towards the end of the list, followed by some recently discovered clusters and groups. Although extensive, the table is probably far from complete.

Table 2. Open clusters




Table 2 (continued)

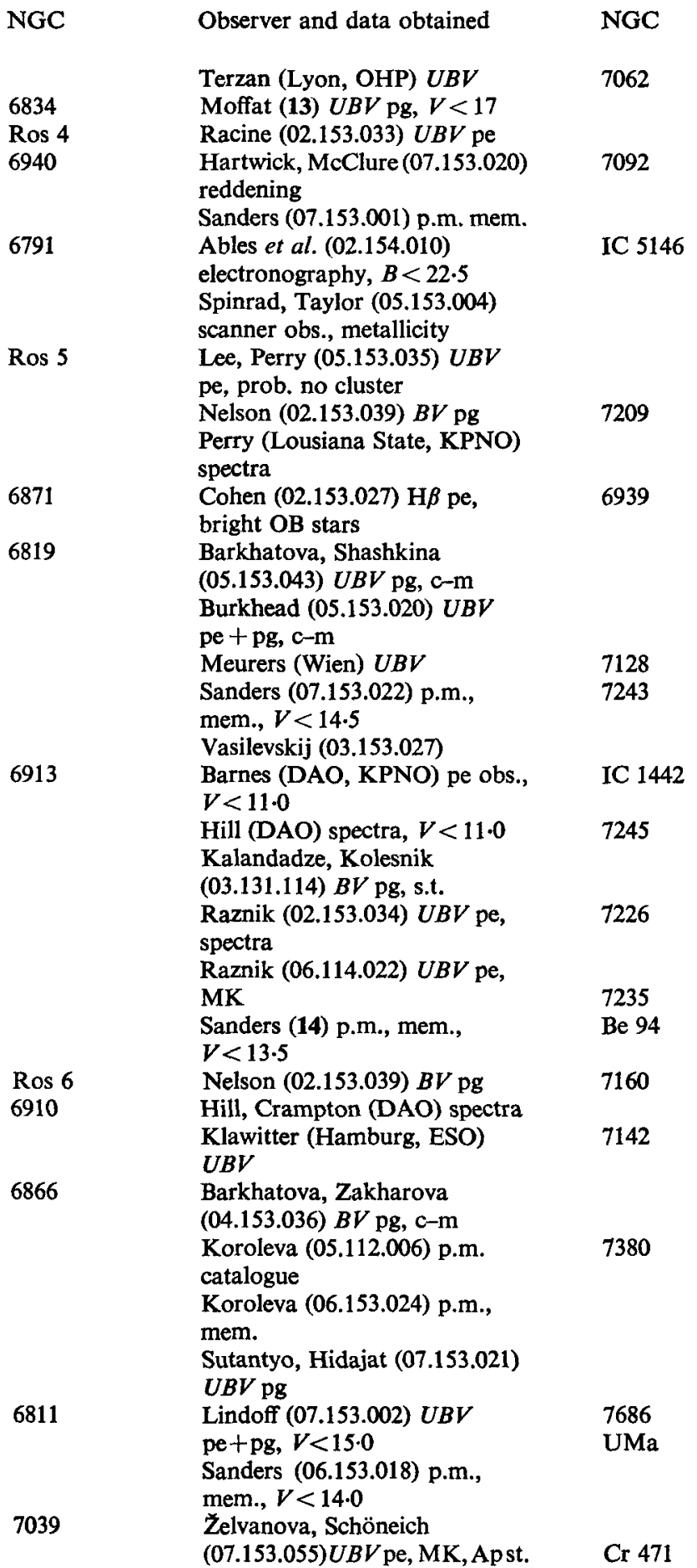

Observer and data obtained

Jones, van Altena (04.153.013)

p.m., mem., $V<14.5$

Sanders (06.153.004) mem.

Artiukhina, Kalinina

(04.112.006) p.m.

Sanders (15) p.m., $V<16.0$

Bernacca, Ciatti (02.153.036)

search Ap st.

Bernacca, Ciatti (5) He weak-

line st.

Samson (Edinburgh) $U B V \mathrm{pg}$, polarization

Artiukhina, Kholopov

(04.154.016) st. counts, $B<15.4$

Sanders (Yerkes) p. m.

Barkhatova, Shashkina (Ural)

phot., c-m

Cannon, Lloyd (02.153.005)

$U B V \mathrm{pg}, \mathrm{c}-\mathrm{m}$

Cannon, Purcell (04.153.041)

p.m., $V<15.5$

Barbon (01.153.010) reddening Hill, Barnes (05.153.012) $U B V$, uvby $\beta, \mathrm{MK}$, r.v.

Koroleva (Pulkovo) p.m.

Yilmaz (04.153.006) $R G U \mathrm{pg}$, c-m

Karaali (07.153.029) UB.V

Yilmaz (04.153.006) RGU pg, c-m

Karaali (07.153.029) $U B V$

Yilmaz (04.153.006) $R G U$ pg, c-m

Moffat (13) $U B V$ pg, $V<17$

Yilmaz (04.153.006) $R G U \mathrm{pg}$, c-m

Conti, van den Heuvel

(04.153.037) MK, r. v., Am st. van den Bergh, Heeringa

(04.153.022) $U B V$ pe + pg, c-m Pendl (Bonn) p.m., $V<15.0$ Sharov (02.153.035) $B V$ pg Ishmukhamedov (02.153.040) p.m., mem.

Moffat (05.153.033) UBV pg, $V<16$

Underhill (01.153.005) r.y., spectr. bin.

Terzan (Lyon, OHP) $U B V$ Crawford, Barnes (01.153.015) uvby $\beta$

Geary, Abt (04.153.008) st. rotation

Akhundova (04.153.031) s.t. 
Table 2 (continued)

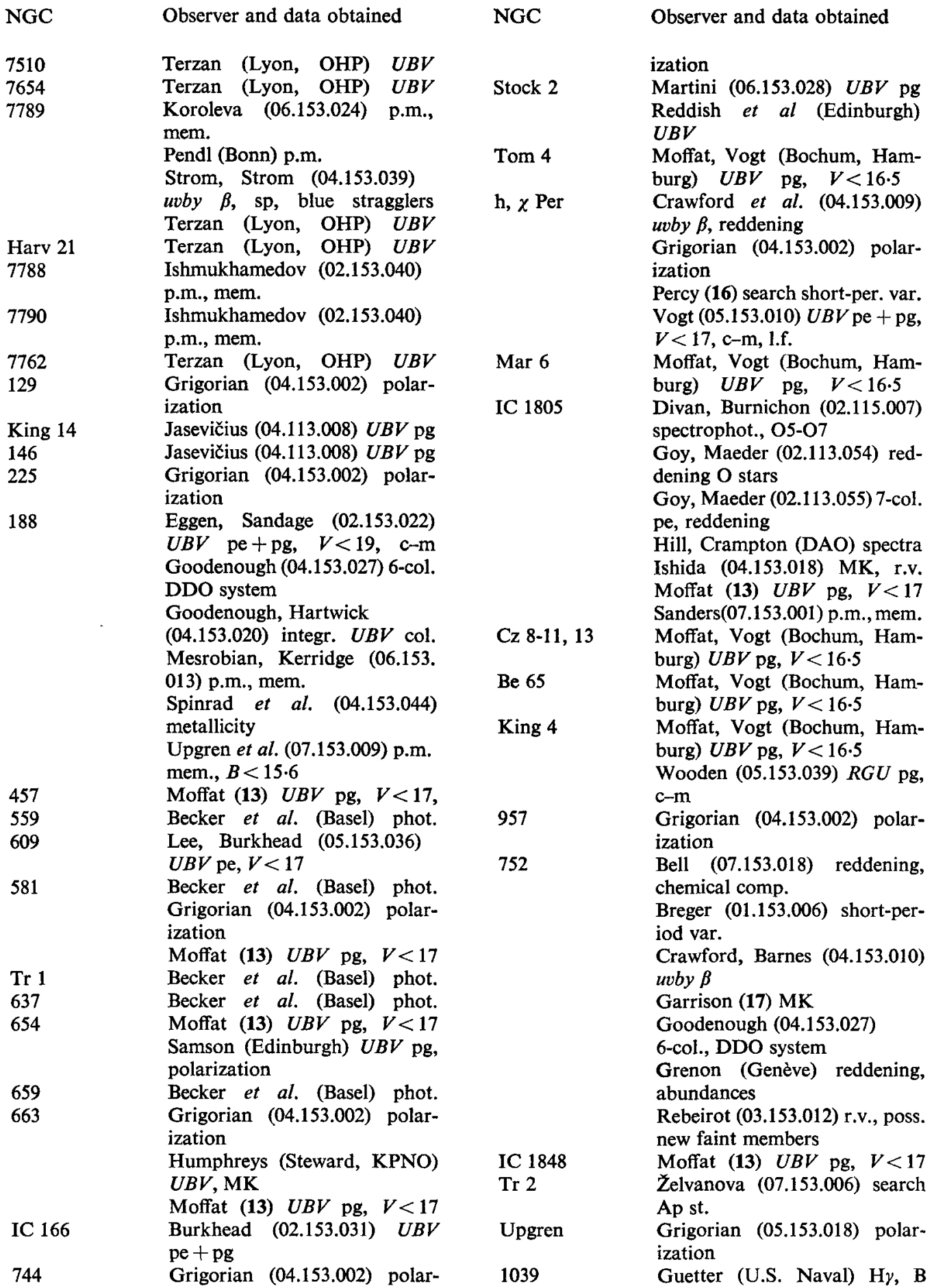


Table 2 (continued)

NGC

Observer and data obtained

and $\mathbf{A}$ st.

Ianna (18) p.m.

Ianna (04.153.019; 06.153.031)

sp., st. rot., Ap st.

Perry (Lousiana State, KPNO)

uvby $\beta$

1502

1245

$\alpha \operatorname{Per}$

IC 361

1528

1513

1545

1342

1605

1664

Be 68

Pleiades

Grigorian (05.153.017) polarimetry

Ishmukhamedov (02.153.040)

p.m.,mem.

Barkhatova, Zakharova (Ural)

phot., c-m, 1.f.

Pendl (Bonn) p.m.

Crawford, Barnes (02.153.014)

uvby $\beta$

Crawford et al. (KPNO) woby $\beta$

Dieckvoss (Hamburg) p.m., expansion, conv. point

Eaton (Dyer) uvby, rotation

effects

Morgan et al. (05.153.006)

MK, HR

Petrie, Heard (04.153.043) r.v., luminosities

Burkhead (Goethe Link) photometry

Barkhatova et al. (03.153.026)

Jones (Lick) p.m.

Barkhatova et al. (03.153.026)

Barkhatova et al. (03.153.026)

Grigorian (04.153.001) polarization

Fang (03.153.009) $R G U$ pg, c-m Fang (03.153.009) $R G U \mathrm{pg}, \mathrm{c}-\mathrm{m}$ Kerridge, Mesrobian (Van

Vleck) p.m.

Wagner (06.153.005) $R G U \mathrm{pg}$ Breger (19) search pulsating var. Crawford et al. (KPNO) uvby $\beta$ Eichhorn et al. (03.153.006) positions, p.m.

Goodenough (04.153.027)

6-col., DDO system

Guetter (U.S. Naval) Hy, B and $\mathbf{A}$ st.

Hesser, Henry (06.113.053)

$\mathrm{K}$-line phot.

Iriarte Erro (03.153.005;

03.153.023) UBVRIJHKL pe, to $\mathrm{GO} \mathrm{V}$

Jones (03.153.001) p.m., orbits, $V<14.0$

Jones (07.153.004) lower main seq., c-m
1857

NGC

1893

2281

Hyades

1647
Observer and data obtained

Kholopov, Artiukhina

(06.153.014) I.f.

Kraft, Greenstein (01.153.029)

$\mathrm{K}$-line em., $V<13$

McCarthy (01.153.030) image-

tube sp., late-type st.

Pesch (Warner and Swasey, KPNO) phot.

Straižys et al. (04.113.059)

UPXYZVS pg

Zappala (07.153.008) Li abundance

Meurers (Wien) $U B V$

Cuffey (New Mexico State, KPNO) $U B V$ pe + pg

Grigorian (04.153.001) polarization

Moffat (13) $U B V \mathrm{pg}, V<17$

Tsarevskij,Abakumov(Baldone) $U B V \mathrm{pg}$

Sanders (06.153.004) mem.

van Altena (01.153.020) p.m., mem., $B<\mathbf{1 8 . 0}$

Breger (04.153.040) short-per.

var., Am st.

Eggen (02.153.029) $R I$ pe, distance

Eggen (Mt Stromlo) UBVRI

Goodenough (04.153.027)

6-col., DDO system

Guetter (U.S. Naval) $\mathrm{H} \gamma, \mathbf{F}-\mathrm{K}$

st.

Hanson (Lick) p.m.

Heintz (02.153.032) dynam.

par., binaries

Hesser, Henry (06.113.053)

$\mathrm{K}$-line phot.

Klemola (Lick) trig. par.

Luyten (05.153.024) catalogue, 4578 st.

Mannery, Wallerstein

(06.153.017) $B V r$ pe

Nissen (04.153.007) $\mathrm{Fe} / \mathrm{H}$ ratio

Pesch (Warner and Swasey,

KPNO) phot.

Sears, Whitford (01.153.007)

6-col., pe

Williams (06.114.088) narrow-

band ind., $\mathrm{Na}, \mathrm{Mn}$ abund.

Wilson (03.114.067) H, K em.

fluxes

Zappala (07.153.008) Li abundance

Hassan (20) $U B V \mathrm{pg}+\mathrm{pe}$, 
Table 2 (continued)

NGC

1807

1817

IC 2157

2168

1662

2175

2169

2141

2420

$\operatorname{Tr} 5$

2264
Observer and data obtained

$V<15 \cdot 7$

Terzan (Lyon, OHP) UBV

Kuz'mina (Ural) $U B V$ pg

Meurers (Wien) $U B V$

Tsarevskij, Abakumov

(Baldone) $U B V \mathrm{pg}$

Kuz'mina (Ural) $U B V$ pg

Becker et al. (Basel) phot.

Tsarevskij, Abakumov

(Baldone) $U B V \mathrm{pg}$

Cudsworth (05.153.038) p.m.,

mem., $B<15.0$

Sanders (06.153.004) mem.

Hassan (07.153.017) $U B V$

pg + pe, $V<15$

Johansen, Gyldenkerne

(03.113.024) 4-col., $\beta$ ind., early A-st.

Schmidt-Kaler, Klinkmann

(Bochum, ESO) $U B V$ pe

Terzan (Lyon, OHP) UBV

Perry (Lousiana State, KPNO) uvby $\beta$

Hill, Crampton (DAO) spectra

Burkhead et al. (21) UBV

pe + pg, $V<18.5$

van Altena, Jones (04.153.005)

p.m., mem., $V<13.5$

Cannon, Lloyd (04.153.014)

p.m., $B V$ pg + pe, $B<16$

Falk (Yale) pg photometry

McClure (Yale) metallicity

Sanders (06.153.004) mem.

Dow, Hawarden (04.153.048)

$B V, V<17, \mathrm{c}-\mathrm{m}$

Bernacca, Ciatti (02.153.036)

search Ap st.

Bernacca, Ciatti (5) search $\mathrm{He}$ weak-line st.

Breger (07.153.007) light var., shells, pulsation

Breger, Dyck (07.132.035) polarization, shells

Guetter (U.S. Naval) $\mathrm{H} \gamma$, B and $\mathbf{A}$ st.

Koch (Flower and Cook,

KPNO) search close bin.

Nandy (05.113.056) UBV ex-

tended to $R I$, pg

Reddish et al. (Edinburgh) $U B V$

Rigel, Crutcher (07.153.012)

$\mathrm{OH}$ obs.

Schmidt (22) optical obs. of
NGC

2251

2254

2236

Praesepe

2244

Cr 107

Orion neb. cl.

Cr 110

Biu 10

2232

M67
Observer and data obtained

IR source

Smith (7) circumstellar gas em.

Strom, Strom (N.Y. State,

KNPO) image-tube sp.

Strom et al. (05.153.026) shells

Strom et al. (07.153.003) IR

and optical obs., shells

Walker (07.114.122) UV-excess

st., sp.

Zappala (07.153.008) Li abundance

Tsarevskij, Abakumov

(Baldone) UBV pg

Kiral (01.153.013) $R G U \quad$ pg

Rahim (04.153.023) RGU pg

Artiukhina (06.153.027) p.m., pg $<16.6$

Breger (04.153.040) short-

period var.

Crawford, Barnes (02.153.004)

uvby $\beta$

Fredrick et al. (Leander McCormick, KPNO) uvby, $\quad V<15$

Guetter (U.S. Naval) $\mathbf{H} \gamma, \mathbf{F}-\mathbf{K}$ stars

Johansen, Gyldenkerne

(03.113.024) 4-col., $\beta$ ind., early A-st.

Jones (05.153.037) p.m., kinematics, $B<12.5$

Zappala (07.153.008) Li abundance

Dufour Lee (03.132.018) $\mathrm{H} \beta, \gamma$ neb. int., $R$ value

Heiser (Dyer, KPNO) H $\beta$

Menon (02.153.015) 21-cm obs., distance

Isserstedt, Schmidt-Kaler (07.152.008) $U B V \mathrm{pg}, V<13$

Abt et al. (04.152.007) st. rotation

Ferrari d'Ochieppo, Göbel

(02.151.066) p.m., mem.

Schild, Chaffee (06.114.096)

MK, energy distr., B st.

Walker (07.114.122) UV-excess

st, sp.

Tsarevskij, Abakumov

(06.153.007) UBV pg, c-m

Vogt (Bochum, ESO) $U B V$ pe Clariá (07.153.026) $U B V \beta$ pe, $V<11.4$

Bond, Perry (06.153.022) uvby, HB st. 
Table 2 (continued)

NGC

2302

Coma

2335

2343

2204

Haff 8

Haff 6

2548

2374

2360

2423

2506

2422

2414

2539

2383

2384

2432
Observer and data obtained

Eggen (07.113.007) UBVRI pe Goodenough (04.153.027)

6-col., DDO system

Goodenough, Hartwick

(04.153.020) integr. $U B V$ col.

Grenon (Genève) reddening, abundances

Griffin, Gunn (Cambridge,

Palomar) r.v., $V<13.0$

Jones (Yale) metallicity

Racine (06.153.009) $U B V$

$\mathrm{pg}+\mathrm{pe}, V<22$

Sanders (15) p.m., $B<16.5$

Sanders (05.153.009) mem.

Spinrad et al. (04.153.044) metallicity

Strom et al. (06.153.029) uvby $\beta$, HB st.

Vogt (Bochum, ESO) UBV pe

Argue, Kenworthy (02.153.025)

$U B V I \mathrm{pg}, B<14$, p.m.

Breger (01.153.006) short-period var.

Chaffee (06.114.104) Fe abun-

dance

Crawford, Barnes (01.153.015)

uvby $\beta$

Johansen, Gyldenkerne

(03.113.024) 4-col., $\beta$ ind., early A-st.

Nissen (04.153.007) $\mathrm{M} / \mathrm{H}$ ratio

Seggewiss (06.113.036) UBV pe,

$V<14 \cdot 3$

Seggewiss (06.113.036) $U B V$ pe,

$V<14 \cdot 3$

(Cape) pe seq., pg plates

Fenkart et al. (23) UBV

Fenkart et al. (23) UBV

Terzan (Lyon, OHP) UBV

Fenkart et al. (23) UBV

McClure (07.153.011) redden-

ing, metallicity

Pendl (Bonn) p.m.

Pendl (Bonn) p.m.

Grigorian (04.153.001) polarization

Ishmukhamedov (02.153.040)

p.m., mem.

Terzan (Lyon, OHP) UBV

Vogt, Moffat (24) $U B V \beta$ pe

Terzan (Lyon, OHP) UBV

Vogt, Moffat (24) $U B V \beta$ pe

Vogt, Moffat (24) $U B V \beta$ pe

Lindoff (Lund, ESO) $U B V$
2367

NGC

2421

2362

$\operatorname{Tr} 7$

Rup 46

2243

Rup 18

Haff 16

Rup 20, 36

$\operatorname{Tr} 9$

2453

Rup 49

2527

Haff 15

Rup 47

2587

2580

2451

Rup 59

2477

2658

2635

Pi 5

Rup 154

Mel 66

Rup 64
Observer and data obtained

pe + pg, $V<15$

Vogt, Moffat (24) $U B V \beta$ pe

Lindoff (Lund, ESO) $U B V$

pe $+\mathrm{pg}, V<16$

Bernacca, Ciatti (02.153.036)

search Ap st.

Bernacca, Ciatti (5) search $\mathrm{He}$ weak-line st.

Goodenough (04.153.027)

6-col., DDO system

Vogt, Moffat (24) $U B V \beta$ pe

Vogt, Moffat (24) $U B V$ pe

(Cape) photometric study

Vogt (Bochum, ESO) UBV

Vogt, Moffat (24) UBV

Vogt, Moffat (24) UBV $\beta$

Pismis (04.153.045) $U B V$ pg + pe, $V<16$

Vogt, Moffat (24) $U B V \beta$ pe Lindoff (Lund, ESO) $U B V$ pe + pg, $V<15$

Seggewiss (06.113.036) $U B V$ pe, $V<13.9$

Vogt, Moffat (24) $U B V \beta$ pe Lindoff (Lund, ESO) UBV pe + pg, $V<14.3$

Vogt, Moffat (24) $U B V$ pe Vogt, Moffat-(24) UBV $\beta$ pe Lindoff (Lund, ESO) $U B V$ pe + pg, $V<15$

Lindoff (Lund, ESO) $U B V$ pe + pg, $V<15$

Goodenough (04.153.027)

6-col., DDO system

Vogt, Moffat (24) $U B V \beta$ pe

Falk (Yale) metallicity

Goodenough (04.153.027)

6-col., DDO system

Hartwick, McClure (07.153.020) reddening

Hartwick et al. (07.153.027)

$U B V$ pe $+\mathrm{pg}$, interm. band phot. giants

Lindoff (Lund, ESO) UBV pe + pg, $V<17$

Vogt, Moffat (24) $U B V$ pe Vogt, Moffat (25) UBV pe, no cl.

Schmidt-Kaler, Klinkmann (Bochum, ESO) UBV pe

(Cape, Radcliffe) pe seq., $V$ $<17.6$

Starischka (Santiago, CTIO) $U B V \mathrm{pe}+\mathrm{pg}$ 
Table 2 (continued)

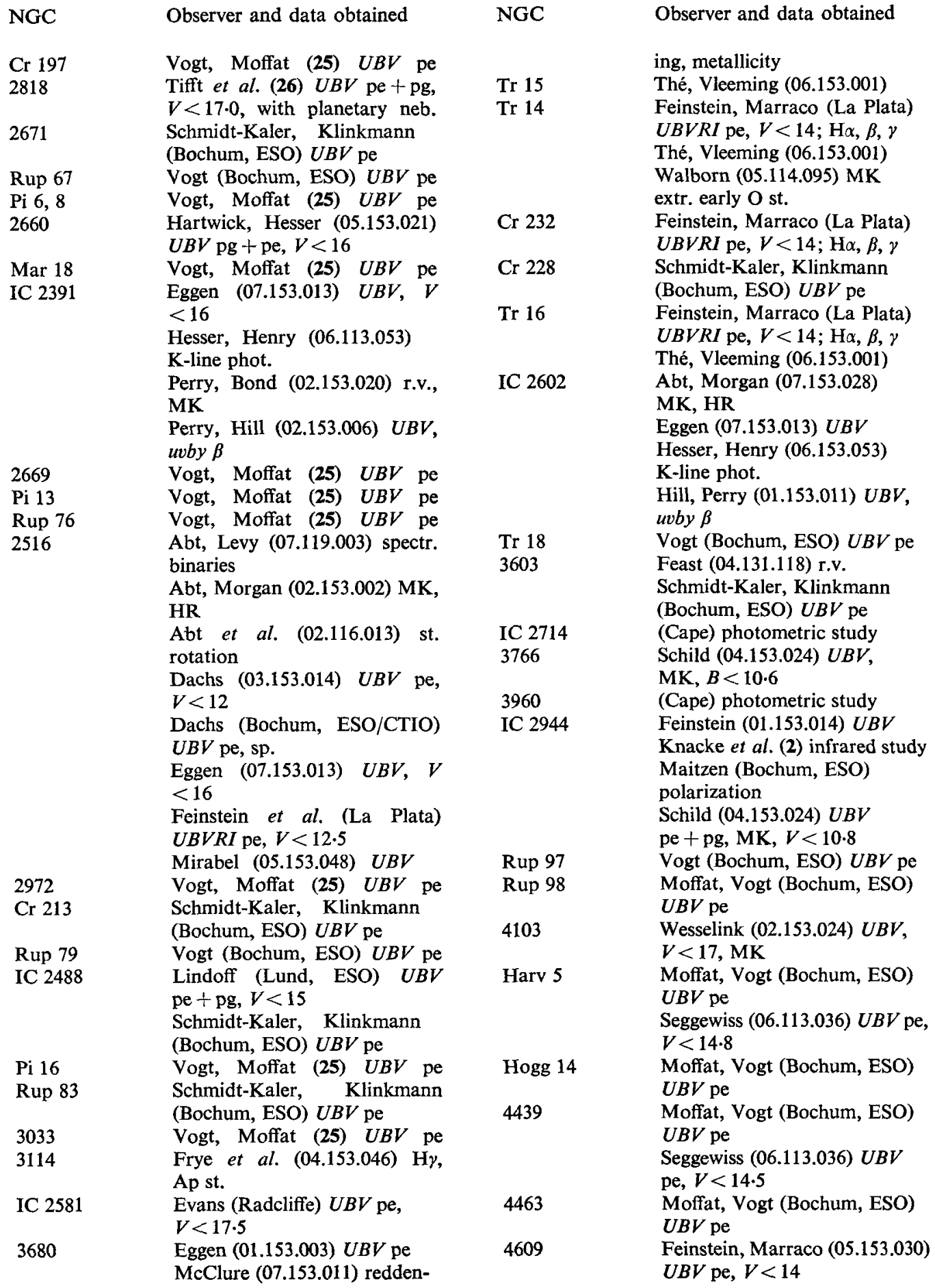


Table 2 (continued)

\begin{tabular}{|c|c|c|c|}
\hline NGC & Observer and data obtained & NGC & Observer and data obtained \\
\hline Hogg 15 & $\begin{array}{l}\text { Feinstein, Marraco (05.153.030) } \\
U B V \text { pe, } V<14\end{array}$ & Rup 118 & $\begin{array}{l}\text { Moffat, Vogt (Bochum, ESO) } \\
U B V \text { pe }\end{array}$ \\
\hline \multirow[t]{2}{*}{4755} & $\begin{array}{l}\text { Dachs (Bochum, ESO/CTIO) } \\
U B V \text { pe, sp. }\end{array}$ & Rup 119 & $\begin{array}{l}\text { Moffat, Vogt (Bochum, ESO) } \\
U B V \text { pe, no cl. }\end{array}$ \\
\hline & $\begin{array}{l}\text { Landolt (Lousiana State, CTIO) } \\
U B V \mathrm{pg} \\
\text { Schild (04.153.024) UBV pe, } \\
\text { MK, } B<10.0\end{array}$ & 6208 & $\begin{array}{l}\text { Eggen (Mt Stromlo) } U B V \\
\text { Lindoff (27) } U B V \text { pe }+\mathrm{pg}, \\
V<14.5 \\
\text { Vogt (Bochum. ESO) } U B V \text { pe }\end{array}$ \\
\hline 4815 & $\begin{array}{l}\text { Moffat, Vogt (Bochum, ESO) } \\
U B V \text { pe, prob. no cl. }\end{array}$ & 6134 & $\begin{array}{l}\text { Lindoff (27) } U B V \text { pe }+ \text { pg, } \\
V<14.5\end{array}$ \\
\hline 4852 & $\begin{array}{l}\text { Lindoff (Lund, ESO) } U B V \\
\mathrm{pe}+\mathrm{pg}, V<15\end{array}$ & 6193 & $\begin{array}{l}\text { Havlen (ESO) UBV uvby } \beta \text {, sp. } \\
\text { Moffat, Vogt (Bochum, ESO) }\end{array}$ \\
\hline Harv 8 & Vogt (Bochum, ESO) $U B V$ pe & & $U B V$ pe \\
\hline Rup 107 & Vogt (Bochum, ESO) $U B V$ pe & 6178 & Moffat, Vogt (Bochum, ESO) \\
\hline Rup 166 & $\begin{array}{l}\text { Moffat, Vogt (Bochum, ESO) } \\
U B V \text { pe, prob. no cl. }\end{array}$ & Hogg 22 & $\begin{array}{l}\text { UBV pe } \\
\text { Moffat, Vogt (Bochum, ESO) }\end{array}$ \\
\hline \multirow[t]{2}{*}{$\operatorname{Cr} 271$} & Moffat, Vogt (Bochum, ESO) & & $U B V$ pe \\
\hline & $U B V$ pe & 6204 & Moffat, Vogt (Bochum, ESO) \\
\hline \multirow{2}{*}{ Hogg 16} & Moffat, Vogt (Bochum, ESO) & & $U B V$ pe \\
\hline & $U B V$ pe & 6169 & Moffat, Vogt( Bochum, ESO) \\
\hline 5138 & $\begin{array}{l}\text { Lindoff (27) } U B V \text { pe }+\mathrm{pg} \\
V<14\end{array}$ & West 1 & $\begin{array}{l}U B V \text { pe, no cl. } \\
\text { Borgman et al. (03.153.011) }\end{array}$ \\
\hline $\operatorname{Tr} 21$ & $\begin{array}{l}\text { Moffat, Vogt (Bochum, ESO) } \\
U B V \text { pe }\end{array}$ & & $\begin{array}{l}\text { IR phot. } \\
\text { Westerlund (ESO) pe + pg }\end{array}$ \\
\hline 5168 & $\begin{array}{l}\text { Moffat, Vogt (Bochum, ESO) } \\
U B V \text { pe }\end{array}$ & IC 4651 & $\begin{array}{l}\text { Eggen }(05.153 .028) U B V \\
\text { pe }+ \text { pg, } V<13\end{array}$ \\
\hline Rup 108 & $\begin{array}{l}\text { Moffat, Vogt (Bochum, ESO) } \\
U B V \text { pe }\end{array}$ & & $\begin{array}{l}\text { Lindoff (27) } U B V \text { pe }+\mathrm{pg} \\
V<14 \cdot 3\end{array}$ \\
\hline 5281 & $\begin{array}{l}\text { Moffat, Vogt (Bochum, ESO) } \\
U B V \text { pe }\end{array}$ & 6249 & $\begin{array}{l}\text { Moffat, Vogt (Bochum, ESO) } \\
U B V \text { pe }\end{array}$ \\
\hline Ly 1,2 & $\begin{array}{l}\text { Schmidt-Kaler, Klinkmann } \\
\text { (Bochum, ESO) } U B V \text { pe }\end{array}$ & $\begin{array}{l}6259 \\
6231\end{array}$ & $\begin{array}{l}\text { (Cape) photometric study } \\
\text { Bessell (Mt Stromlo) sp. }\end{array}$ \\
\hline 5606 & $\begin{array}{l}\text { Moffat, Vogt (Bochum, ESO) } \\
U B V \text { pe }\end{array}$ & & $\begin{array}{l}\text { OB st. } \\
\text { Crawford et al. }(06.153 .025)\end{array}$ \\
\hline & Clariá (06.153.008) $U B V \beta$ pe & & $u v b y \beta$ \\
\hline 5662 & $\begin{array}{l}\text { Moffat, Vogt (Bochum, ESO) } \\
\text { UBV pe }\end{array}$ & & $\begin{array}{l}\text { Schild et al. (01.152.004) UBV } \\
\text { pe, MK }\end{array}$ \\
\hline Pi 21 & $\begin{array}{l}\text { Schmidt-Kaler, Klinkmann } \\
\text { (Bochum, ESO) } U B V \text { pe }\end{array}$ & 6242 & $\begin{array}{l}\text { Moffat, Vogt (Bochum, ESO) } \\
U B V \text { pe }\end{array}$ \\
\hline Pi 20 & $\begin{array}{l}\text { Moffat, Vogt (Bochum, ESO) } \\
U B V \text { pe }\end{array}$ & $\begin{array}{l}\text { Rup } 127 \\
\text { Pi } 24\end{array}$ & $\begin{array}{l}\text { Vogt (Bochum, ESO) UBV pe } \\
\text { Moffat, Vogt (Bochum, ESO) }\end{array}$ \\
\hline \multirow[t]{4}{*}{5823} & Cullmann (Würzburg, ESO) & & $U B V$ pe \\
\hline & $U B V$ pe $+\mathrm{pg}, V<17$ & 6396 & Vogt (Bochum, ESO) $U B V$ pe \\
\hline & Lindoff (Lund, ESO) $U B V$ & 6374 & Antalová (07.113.010) UBV pg \\
\hline & pe + pg, $V<15$ & 6383 & Antalová (07.113.010) $U B V \mathrm{pg}$ \\
\hline \multirow[t]{2}{*}{5822} & $\begin{array}{l}\text { Hartwick, McClure (07.153.020) } \\
\text { reddening }\end{array}$ & 6475 & $\begin{array}{l}\text { Abt, Jewsbury }(01.153 .024) \\
\text { st. rotation }\end{array}$ \\
\hline & McClure (Yale, CTIO) $U B V$ & & Abt et al. $(03.153 .003)$ spectr. \\
\hline 6025 & $\begin{array}{l}\text { Feinstein (06.153.030) } U B V R I \\
\text { pe, } V<13.5\end{array}$ & & $\begin{array}{l}\text { binaries } \\
\text { Constantine } \text { et al. }(01.153 .027)\end{array}$ \\
\hline Ly 4 & Vogt (Bochum, ESO) UBV pe & & p.m. \\
\hline 5999 & $\begin{array}{l}\text { Lindoff (Lund, ESO) } U B V \\
\text { pe }+p g, V<14.5\end{array}$ & & $\begin{array}{l}\text { Conti, van den Heuvel } \\
(04.153 .037) \mathrm{MK} \text {, r.v., Am st. }\end{array}$ \\
\hline Ly 6 & Vogt (Bochum, ESO) UBV pe & & Hesser, Henry (06.113.053) \\
\hline
\end{tabular}


Table 2 (continued)

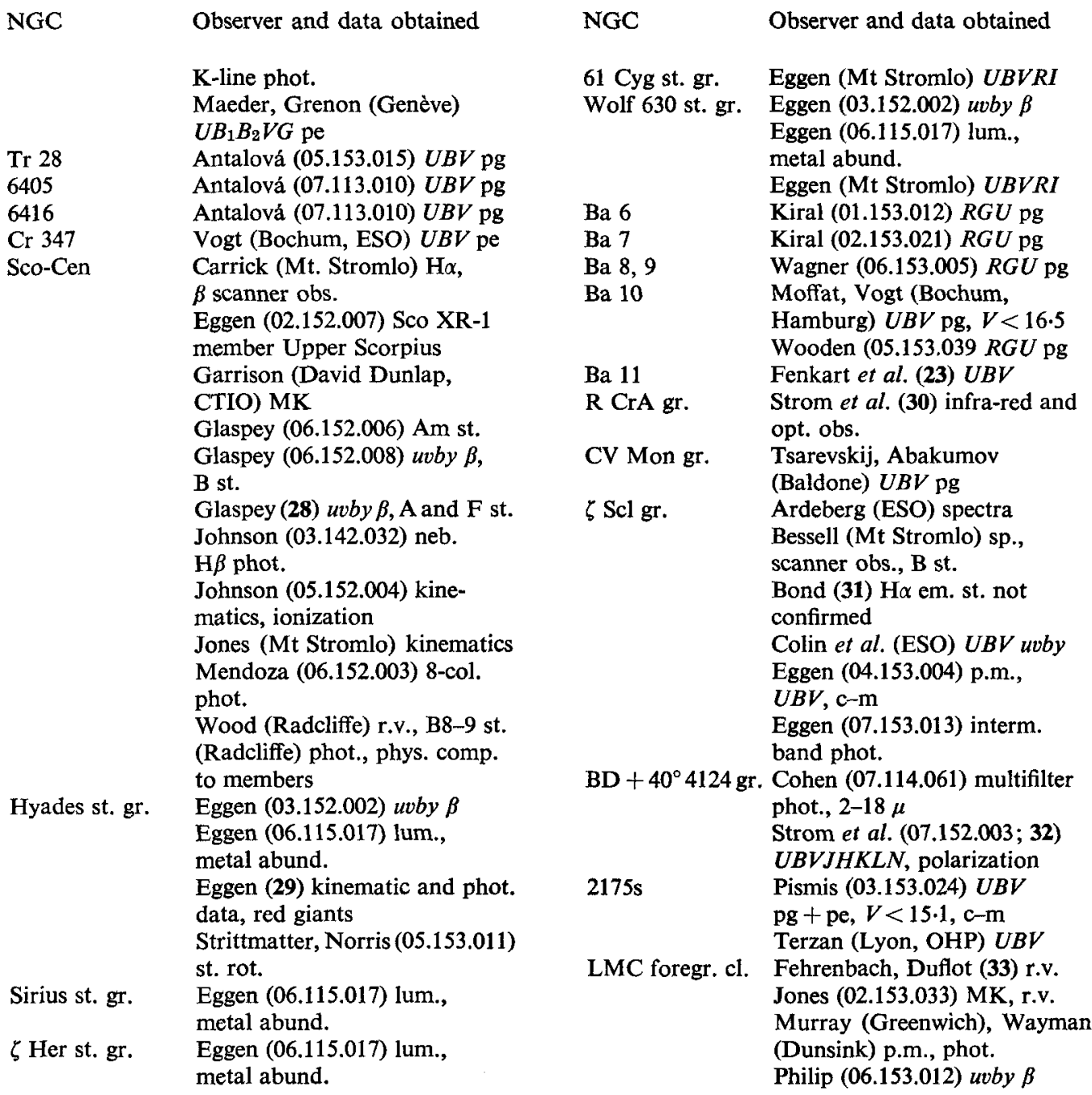

Abbreviations: cl. = cluster, $\mathrm{c}-\mathrm{m}=$ colour-magnitude diagram, $\mathrm{HB}=$ horizontal branch, $\mathrm{HR}=\mathrm{Herztsprung}$ Russell diagram, 1.f. = luminosity function, mem. = membership, $\mathbf{M K}=\mathbf{M K}$ classification, p.m. = proper motion, sp. = spectra, st. = stars (or stellar), s.t. = low-dispersion spectral types.

The importance of proper motions and radial velocities for discriminating between field stars and physical members of open clusters have become increasingly apparent. Many membership studies from relative proper motions are listed in Table 2. Several of these are based on plates taken with the Yerkes refractor. Other extensive proper-motion programmes are in progress at Lick and at Pulkovo. The development by Griffin (03.112.005) of the photoelectric technique for radial-velocity measurements holds great promise for the future. Griffin and Gunn have recently used the instrumentation on the Hale telescope and have measured radial velocities for many stars in $M 67$, down to $V=13.0$. The accidental standard errors are about $0.5 \mathrm{~km} \mathrm{~s}^{-1}$. There are small but significant differences among the velocity values obtained for individual stars, indicating directly the presence of a velocity dispersion in the cluster. 
That observed well-behaved sequences in the $U B V$ and similar photometric systems are not sufficient for proving the physical reality of a cluster, is shown by Bürki (34) and by Bürki and Maeder (35). From the application of new well-defined photometric criteria, they question the physical reality of several groupings, which have generally been considered as real clusters.

Studies of the frequency of binaries and of stellar rotational velocities in clusters have primarily been made by Abt and collaborators. Both binary frequency and mean rotational velocity vary from cluster to cluster. The observational data pertaining to stellar rotation and the probable explanations of the differences found among clusters were reviewed by Abt (04.153.021). Maeder (36) considered in detail the effects of stellar rotation on the $c-m$ diagrams, and discussed in a separate paper (05.153.002) the influence of rotation on age estımates. Existing rapid and uniform rotation, if neglected, is found to lead to large overestimates, amounting to $60-70 \%$ for the Pleiades and $\alpha$ Per clusters. Hazlehurst and Thomas (04.153.015) tried to disentangle the rotational effects for the brighter Pleiades individually, while Smith (05.153.014) analysed the Praesepe stars and the Hyades statistically.

Considerable evidence has accumulated showing that there are differences in the metal abundance for the various open clusters, which are not strictly a function of age, as would follow from gradual metal-enrichment of our Galaxy. From photometry of a group of faint metallic lines in F stars, Nissen (04.153.007) found a surprisingly high $\mathrm{Fe} / \mathrm{H}$ ratio for the Hyades. A model-atmosphere analysis by Chaffee et al. (05.153.032) based on medium-dispersion spectrograms gave an $\mathrm{Fe} / \mathrm{H}$ ratio that is $50 \%$ higher for the Hyades than for the Pleiades, while Gustafsson and Nissen (07.064.043) find that the difference amounts to $80 \%$. The Coma cluster and the Ursa Major moving cluster are photometrically similar to the Pleiades $(01.153 .015 ; 04.153 .007)$, indicating similar metal abundances.

Still higher metallicities were derived by Spinrad and Taylor from scanner observations for the old clusters M67 and NGC 188 (02.114.115) and for NGC 6791 (05.153.004). The super-metallicity of M67 and NGC 188 was further substantiated by Spinrad et al. (04.153.044). Gottlieb and Bell (37) in an analysis confirmed that the Hyades, M67 and NGC 188 are more metal-rich than the Sun, and Hartwick et al. (07.153.027) found NGC 2477 to have high metallicity.

Eggen and Sandage (02.153.022), on the other hand, concluded from photometric data that M67 and NGC 188 have almost solar metallicities. For M67 the same conclusion is drawn by Barry and Cromwell (38) from a recent preliminary study of image-tube spectrograms of dwarfs and sub-giants. Although the super-metallicity remains controversial, it seems certain that the disk clusters exhibit quite a range in metal abundance, probably due to local enrichment.

Among the many studies of individual clusters listed in Table 2, only few can be explicitly mentioned here. Vogt (05.153.010) made an extensive photographic investigation of $\mathrm{h}$ and $\chi$ Per. For both clusters $\mathrm{c}-\mathrm{m}$ diagrams were obtained by statistical subtraction of field stars. Ages were derived as well as a new determination of the zero-age main sequence. Another new determination of the ZAMS was made by Evans (07.115.018) using his photometric data for NGC 2362 and 3766.

Photographic photometry of M67 to the limit of the Hale telescope, i.e. down to $M_{V}=12$, was performed by Racine (06.153.009). The number of faint blue objects in the field is similar to that in other high-latitude fields, indicating that there are no white dwarfs in the cluster above the limiting magnitude. The white dwarfs in M67 are therefore significantly fainter than those in the Hyades.

Tifft et al. (26) studied NGC 2818, which is interesting because of a planetary nebula in the field. The nebula is of high excitation, and the radial velocites indicate that it is a cluster member. The cluster is somewhat younger than NGC 752.

\section{Galactic globular clusters}

Papers and programmes on galactic globular clusters are listed in Table 3. Studies of surface brightnesses and integral properties are not tabulated, and only few papers on variable stars have been listed. The variables are covered by Dr Sawyer Hogg in an Appendix to the Commission 27 report. 
Table 3. Globular clusters

NGC Observer and data obtained

47 Tuc Cathey (Lick) $U B V$ pe, $B<14.5$

Eggen (07.113.007) UBVRI pe

Goodenough (04.153.027) 6-col., DDO system

McClure, Osborn (Yale) 6-col., DDO system

288 Cannon (Mt Stromlo) pe seq., $V<18$

362 Eggen (07.113.007) UBVRI pe

1261 Alcaino, Contreras (05.154.002) UBV pe + pg, $V<17.6$

1851 Alcaino (06.154.015) $U B V$ pe $+\mathrm{pg}$, $V<16.0$

2808 Alcaino (06.154.015) $U B V \mathrm{pe}+\mathrm{pg}$, $V<16.0$

Philip, Tifft (01.154.008) pe seq. White (03.154.004) $U B V$ pe seq., $V<14.1$

$3201 \quad$ Philip (39) uvby, HB stars

White (05.113.046) UBV pe seq., $V<11.4$

White, Kraft (07.154.018) pseudo c-m

4590 Terzan (Lyon, OHP) $m_{\mathrm{r}}$ seq., $V<16.5$

4833 Alcaino (05.154.014) $\mathrm{BV}$ pe $+\mathrm{pg}$,

$V<16.3$

Menzies (07.154.008) $U B V$ pe + pg,

$V<16.7$

5024 Panova (Pulkovo) p.m., $B<16.0$

$\omega$ Cen Cannon (Mt Stromlo) pe seq., $V<18$

Dickens (40) $\mathrm{CH}$ star, $\mathrm{C}^{12} / \mathrm{C}^{13}$ ratio

Dickens et al. (41) phot., sp., red. var.

Eggen (07.113.007) UBVRI pe

Geyer (07.154.023) $U, U-B, \mathrm{RR}$

Lyr var.

Geyer, Szeidl (03.154.011; 03.154.016)

mean $V, B-V$, ampl. RR Lyr var.

Newell et al. (02.154.011) UBV pe,

BHB st.

Norris (Yale) phot., sp.

Philip (Dudley) $u v b y$, BHB st.

Rodgers (07.114.016) spectra, col.,

BHB st.

Stock, Wing (42) carbon stars

M3 Crawford, Barnes (02.154.005) uvby $\beta$, reddening

Kadla, Spasova (07.154.024) red-giant members

McClure, Racine (02.154.004) reddening

Osborn (07.154.030) 6-col., DDO

system

Philip (Dudley, Steward, KPNO) uvby

$\beta$, BHB st.

Sandage (02.154.003) reddening, $\mathrm{He}$ abund.

Sandage (04.154.017) $U B V \mathrm{pe}+\mathrm{pg}$,
NGC Observer and data obtained

$V<21 \cdot 2, \mathrm{c}-\mathrm{m}$

Simoda, Fukuoka (Tokyo) 1.f.

Strom, Strom (03.154.001) analysis O st.

Zhukov (01.154.004) members

Zhukov (07.154.012) BV pg, p.m.

IC 4499 White (05.113.046) UBV pe seq.,

$V<12.4$

$5897 \quad$ Eggen (07.113.007) UBVRI pe

M5 Coutts et al. (02.154.009) study var. gap

Eggen (07.113.007) UBVRI pe

Kadla, Spasova (07.154.024) red-giant

members

Osborn (07.154.030) 6-col., DDO

system

Simoda, Tanikawa (04.154.002) $B V$

$\mathrm{pg}+\mathrm{pe}, V<15 \cdot 5$, c-m

Simoda, Tanikawa (07.154.002) 1.f., $V<21$

Tifft (Dudley, CTIO) uvby, BHB st.

Zhukov (01.154.004) members

Zhukov (07.154.012) $B V$ pg, p.m.

5927 White (03.154.004) $U B V$ pe seq.,

$V<16.0$

White, Kraft (07.154.018) pseudo c-m

5986 White (05.113.046) $U B V$ pe seq.,

$V<11.4$

6101 White (03.154.004) UBV pe seq., $V<13 \cdot 1$

M4 Eggen (07.113.007) $U B V R I$ pe

Newell (04.154.003) $U B V$ pe, BHB st.

Norris (Yale) phot., sp.

Philip (05.154.003) uvby, BHB st.

6171 Dickens (04.144.015) UBV pg, RR

Lyr var.

Dickens, Rolland (43) UBV pg + pe,

$V<19.0$, c-m

M13 Ables et al. (02.154.010) electronography

Bijaoui (05.154.012; 05.154.013)

electronography

Cathey (Lick) $U B V$ pe

Crawford, Barnes (02.154.006) uvby $\beta$, reddening

Griffin, Gunn (Cambridge, Palomar)

r.v., $V<13.1$

Kadla, Spasova (07.154.024) red-giant

members

McClure, Racine (02.154.004) reddening

Newell, Zinn (Yale, U.S. Naval Obs.

Flagstaff) $U B V$, electronography

Osborn (07.154.030) 6-col., DDO

system

Philip (Dudley) uvby $\beta$, BHB st.

Sandage (02.154.003) reddening, He abund. 
Table 3 (continued)

NGC Observer and data obtained

Sandage (04.154.017) $U B V$ pe + pg, $V<21 \cdot 5, \mathrm{c}-\mathrm{m}$

Simoda, Tanikawa (07.154.002) 1.f., $V<19$

M12 Racine (05.154.007) $B V$ pe + pg,

$V<18 \cdot 5$

6254 Osborn (07.154.030) 6-col., DDO system

6266 White (05.113.046) $U B V$ pe seq., $V<10.7$

6304 Terzan, Rutily (07.154.009) $m_{\mathrm{r}}, m_{\mathrm{ir}}$ pg seq.

6316 White (05.113.046) $U B V$ pe seq., $V<11.5$

M92 Ables et al. (02.154.010) electronography

Cathey (Lick) $U B V$ pe

Eggen (07.113.007) UBVRI pe

Hartwick (04.154.009) l.f.

Kadla (06.154.004) p.m., $V$, red giants,

$V<13.4$

Melnikov, Kadla (07.154.025) non-

member giants

Osborn (07.154.030) 6-col., DDO

system

Sandage (02.154.003) reddening, $\mathrm{He}$

abund.

Sandage (04.154.017) $U B V$ pe + pg,

$V<22 \cdot 3, \mathrm{c}-\mathrm{m}$

Strom, Strom (06.154.003) sp., AB st.

6352 Alcaino (05.154.001) $U B V$ pe + pg,

$V<16.2$

Hartwick, Hesser (07.154.026) UBV

$\mathrm{pe}+\mathrm{pg}, V<19, \mathrm{c}-\mathrm{m}, \mathrm{Fe} / \mathrm{H}$

6362 Alcaino (07.154.001) UBV pe + pg,

$V<15.5$

6388 White $(05.113 .046) U B V$ pe seq., $V<12.8$

White, Kraft (07.154.018) pseudo c-m

M14 Smith (Western Ontario) phot. pg

6401 Terzan, Rutily (07.154.009) $m_{\mathrm{r}}$ pg seq.

6397 Cannon (Mt Stromlo) pe seq., $V<18$
NGC Observer and data obtained

Norris (Yale) phot., sp.

Philip (Dudley) $u v b y \beta$

6522 van den Bergh (06.113.050) $B V$ pe + pg,

$V<16 \cdot 6$, c-m

6528 van den Bergh (06.113.050) $B V$ pe + pg,

$V<17 \cdot 7$, c-m

6541 Alcaino (06.154.002) $U B V \mathrm{pe}+\mathrm{pg}$,

$V<16.9$

6638 Terzan (Lyon, OHP) $m_{r}$ seq.

M69 Evans, Menzies (05.154.016)

M22 Eggen (07.113.007) UBVRI pe

Kadla (Pulkovo) p.m., $B<16 \cdot 6, U B V$

pg, 2000 st.

Kadla (Pulkovo) red-giant members

Norris (Yale) phot., sp.

Strom, Strom (06.154.003) sp., AB st.

6752 Alcaino (07.154.001) $B V$ pe + pg,

$V<15.5$

Cannon (Mt Stromlo) pe seq., $V<18$

Eggen (07.113.007) UBVRI pe

Tifft (Dudley, CTIO) $u v b y \beta$, BHB st.

6809 Philip (04.154.010) woby, BHB st.

6838 Arp, Hartwick (06.154.001) UBV

pe + pg

Meurers, Prochazka (02.112.008) p.m.

Sanders (06.154.012) p.m., mem., $V<15$

6981 Dickens (07.154.021) $U B V$ pe + pg,

$V<18 \cdot 3$

Dickens, Flinn (44) phot. RR Lyr var.

7006 Hartwick, McClure (45) 6-col., DDO

system

M15 Newell, Zinn (Yale, U.S. Naval Obs.

Flagstaff) $U B V$, electronography

Panova (Pulkovo) p.m., $B<16.0$

Sandage (02.154.003) reddening, He

abund.

Sandage (04.154.017) $U B V$ pe + pg,

$V<22 \cdot 0, \mathrm{c}-\mathrm{m}$

M2 Eggen (07.113.007) UBVRI pe

7099 Dickens (07.154.022) $U B V$ pe + pg, $V<17 \cdot 0$

Abbreviations: $\mathrm{AB}=$ asymptotic branch, $\mathrm{BHB}=$ blue horizontal branch, $\mathrm{c}-\mathrm{m}=$ colour-magnitude diagram, $\mathrm{HB}=$ horizontal branch, $1 . \mathrm{f} .=$ luminosity function, mem. $=$ membership, p.m. $=$ proper motion, sp. $=\mathrm{spec}-$ tra, st. = stars.

Photometry in the $u v b y$ and $\beta$ systems of the integrated light for 16 clusters were made by Johnson and McNamara (02.154.006). Nine globulars were measured by Neff (03.158.094) in an intermediate bandpass-system. Guetter et al. (U.S. Naval Observatory, Flagstaff) measured 66 clusters on the Stebbins-Whitford six-colour system, and Faber (07.158.138) measured ten clusters in a 10-colour system.

The ellipticity of $\omega$ Cen was studied by Sisteró and Fourcade (03.154.007). Högner et al. (Pulkovo) made a similar study of M92 by means of 92 equidensity curves in $U B V R$. Work on M3, M13 and M15 is in progress. 
Kuzmin and Veltmann (Tartu) started some years ago photoelectric observations of the brightness distribution, using various filters and tracings with a long and narrow slit. Measurements of M3 and M15 have been published (01.154.010), other clusters observed are M2, M5, M13, and M92.

Another comprehensive programme for determining brightness distributions is being undertaken by Kron et al. (U.S. Naval Observatory, Flagstaff). The clusters are recorded with an electronic camera, using $5 \mathrm{~min}$. as standard exposure time for unreddened objects at the zenith. For other objects the exposure time is prolonged correspondingly, in order to retain uniform detectability out to an isophote of given brightness. This far, five objects (NGC 2419, 4590, 5024, M3, and NGC 5466) have been completed, showing in all cases good agreement with photoelectric results. The new data, however, cover a larger range in radius and have very much more observational weight than the photoelectric data. A representative sample, perhaps all clusters observable from Flagstaff, will be recorded.

Electronography has been used by Bijaoui $(05.154 .012 ; 05.154 .013)$ for studying the stellar distribution in the central part of M13. Determinations of stellar distributions by star counts were made by several authors, for example by Blaghikh (03.154.019) for NGC 5024 and 5053, by Blaghikh and Castellani $(05.154 .008$; 06.154.006) for M13, and by Kholopov (05.154.006) for M56. The possibility of using the Palomar Sky Survey for this purpose was investigated by Castellani et al. (07.154.016). King and Peterson (Berkeley) are collecting extensive data on radii of globular clusters.

Griffin's photoelectric technique for radial velocity measurements proved highly valuable also for the globulars. Using the Hale telescope Griffin and Gunn measured some 60 stars in M13, down to $V=13.1$. The accidental standard error is $1.3 \mathrm{~km} \mathrm{~s}^{-1}$ and the extreme velocity range $20 \mathrm{~km} \mathrm{~s}^{-1}$. An effect due to cluster rotation is apparent.

Terzan (Lyon) is studying a large number of clusters by means of red and infra-red photography, primarily for research on variables. He is confirming that NGC 6256, which is omitted in recent catalogues, is a globular cluster. In addition to previous discoveries of highly obscured clusters, Terzan (05.155.026) reported four new clusters, Terzan 9-12, in the region of the galactic centre. Terzan 11 is, however, identical with Terzan 5, discovered for the second time (07.155.062). The true nature of these objects, especially that of Terzan $5(07.158 .035)$, are not yet definitely settled. Two other reddened objects in the Sagittarius region, reported by Wray $(06.154 .011)$ as probable globular clusters, are identical with Terzan 1 and 2.

\section{Extra-galactic clusters and associations}

Because of their proximity the clusters and associations in the Magellanic Clouds can be studied in more detail than those in other galaxies. A survey of LMC associations was given by Hodge and Lucke $(03.159 .001 ; 04.159 .003)$. Other recent studies of associations in the LMC are by Hodge (04.159.005), Lucke (06.159.011;07.152.005), and by Westerlund (06.159.015). A review of data on the Cloud clusters was given by Gascoigne (06.159.016). The c-m diagrams of individual clusters are studied by Walker using electronographic techniques. Published papers in this programme include Kron 3 (04.159.006), NGC 2209 (05.159.009), NGC 2257 (07.159.011), and NGC 419 (46). Hodge (07.159.005) obtained c-m diagrams for five faint LMC-clusters. Gascoigne and Robertson (Mt Stromlo) are working on young clusters in the LMC. Cepheids were observed in NGC 2136, 2157, 2164, and 2214. C-m diagrams for these clusters, and for NGC 1818, 2004, and 2100, were obtained, mostly by Robertson. The cepheids associated with NGC 2136 are 0.6 mag. too bright for their periods, indicating that the cluster may be some $10 \mathrm{kpc}$ nearer than the LMC. This is supported by spectra obtained by Freeman (Mt Stromlo), showing that the cluster radial velocity differs by some $100 \mathrm{~km} \mathrm{~s}^{-1}$ from $21-\mathrm{cm}$ velocities of surrounding parts of the LMC. Variables in NCG 1466 were studied by Wesselink (05.159.008) from Radcliffe material. Andrews (Radcliffe) completed photographic observations, based on a photoelectric sequence, for the NGC 371 region in the SMC. Feast (47) found several of the bright main-sequence stars in NGC 330 to be of the Be type. Integrated properties of Cloud clusters were studied by Ford (48; 05.159.004), by Andrews and Evans $(06.159 .024 ; 49)$, and by Danziger (Harvard). New six-colour photometry for 15 objects 
was made by Kron et al. Freeman and Gascoigne (05.159.005) measured the radial surface-brightness distribution for NGC 1831 and 1866, and discussed the theoretical implications.

New observations of magnitudes and colours for the brightest globular clusters in the Fornax dwarf galaxy were made by Demers $(01.158 .048)$, by Hodge (02.158.092), and by de Vaucouleurs and Ables (03.158.029). The colours are normal and match the bluest globulars in the Galaxy. Intermediate-band photometry for some of these clusters was obtained by Danziger.

Scanner abundance-studies of the clusters $\mathrm{H} \mathrm{87,} \mathrm{B} \mathrm{282,} \mathrm{and} \mathrm{M} \mathrm{II} \mathrm{in} \mathrm{M31} \mathrm{were} \mathrm{published} \mathrm{by}$ Spinrad and Schweizer (07.154.006). The authors confirm previous observations by van den Bergh (02.158.077), who found these clusters to be quite strong-lined. Six-colour data for 7 globulars in M31 were obtained by Kron et al. Sharov (Moscow) continued a study of the globulars in this galaxy and discovered several objects at large distances from the centre. New OB associations in the outer region of the system were found by Richter (06.158.007).

Integrated photometry of bright associations in NGC 6946 was reported by Khachikian and Sahakian (04.158.062). Van den Bergh (05.158.078) found that the nuclear region of M82 contains a number of super-clusters with $M_{V} \approx-15$. He suggests that the formation of these was triggered by the explosion that occurred in M82 about $10^{7}$ yr ago. These super-clusters are not related to the super-associations that Shahbazian (04.158.084) has studied in several galaxies and which are situated in the spiral arms.

Hanes and Racine (06.154.008) completed an $U B V$ photometric study of the integrated light of nearly 1000 globular clusters in M87. The colours are found to be normal and the colour dispersion the same as for galactic globulars, suggesting that the metallicity variations in the halo of M87 are similar to those in the halo of our Galaxy. A more detailed spectrophotometric analysis of some few clusters is attempted by Racine and Oke. Broad-band photometry and luminosityfunction investigations are being extended to many Virgo globular systems by Racine and Hanes. Preliminary results indicate that the apparent luminosity-function reaches a limit at $B \approx 21.0$ for all giant ellipticals in Virgo.

De Vaucouleurs (03.158.030) investigated the possible use of the brightest clusters in galaxies as distance indicators.

\section{STRUCTURE AND DYNAMICS OF ASSOCIATIONS}

The term association is vaguely defined and is used for stellar aggregates covering large ranges in size, mass and luminosity. Super-associations, easily observable in remote galaxies, have $M_{V} \approx-15(04.158 .084)$, while the small $\mathrm{OB}$ associations in our Galaxy are about 10 magnitudes fainter. Objective-prism surveys often seem to disclose loose groupings of stars, noticed because of similarity in spectral type and magnitude. Lodén (Uppsala) is preparing lists of such groupings, which may in some cases represent highly diluted clusters or associations. As an obvious example a small group of B stars with peculiar spectra was reported (06.114.070).

Among the recent structural studies of recognized associations those of Sco OB 1 by Laval $(06.152 .004 ; 07.152 .007 ; 07.152 .014 ; 9)$ are remarkable. There are about 150 stars earlier than B6 in Sco OB 1, and photometrically they all seem to form a physical group at a distance of $2 \mathrm{kpc}$. Kinematical data show, however, that the association consists of a southern and a northern subgroup. The southern one, with mean radial velocity $-28.7 \mathrm{~km} \mathrm{~s}^{-1}$, includes the open cluster NGC 6231. This sub-group, which is relatively dense and probably expanding, contains supergiants, emission-line stars and two Wolf-Rayet stars. The northern group has mean velocity $-23.6 \mathrm{~km} \mathrm{~s}^{-1}$, it is more sparse, and lack of data leave the question of expansion open. It contains three highly reddened O-stars and is responsible for the ionization of the large nebula IC 4628 . A similar division of the region is implied by the distribution of the ionized gas, the corresponding mean radial-velocities for the gas components are -29.7 and $-20.2 \mathrm{~km} \mathrm{~s}^{-1}$. The gas density in the southern group is much lower than in the northern, and IC 4628 itself seems to consist of five dense condensations. This highly unstable situation, a high-density $\mathrm{H}$ in region immersed in lowdensity surroundings, indicates that the northern group is younger than the southern. Careful 
examination of the photometric data supports this view. A photometric age of $5 \times 10^{6} \mathrm{yr}$ is found for the southern group, while its kinematical age, on the assumption of linear expansion, is $0.9 \times 10^{6}$ yr. Laval finds it even possible to trace the propagation of star formation within both sub-groups. In each case star formation seems to have progressed in the direction of galactic rotation, i.e. in the direction opposite to that expected, if gravitational contraction is supposed to be triggered by a density wave travelling with a velocity lower than the circular one.

Garmany (1) studied the internal motions in Cep OB 3, which contains two sub-groups in much the same way as Sco OB 1, i.e. a younger group associated with nebulosity and an older, more dispersed group. The two sub-groups are found to expand from each other in galactic longitude, and from this Garmany finds a kinematic age of $5 \times 10^{5} \mathrm{yr}$. Lesh (02.152.002) derived the kinematic age $1.3 \times 10^{6} \mathrm{yr}$ for Per OB 2 and $2.5 \times 10^{6} \mathrm{yr}$ for the concentrated sub-group of Lac OB 1 . The dispersed sub-group of Lac OB 1 appears to have 1 mag. smaller distance modulus than the concentrated group.

Vasilevskis (06.132.003) emphasized that the expansion found by Strand in 1958 for the Orion Nebula cluster (sub-group d of Ori $O B$ 1) is spurious and due to instrumental scale-changes. There is no observational proof for the expansion of this group at present.

The distribution of space velocities of $\mathrm{O}$ and $\mathrm{B}$ stars in a synthetic association was studied by Mirzoyan and Mnatsakanyan (04.152.008), and a method was developed for determining the mean space-velocity as function of distance from the centre of a spherical system. Application of this method to the synthetic association indicates expansion and confirms the dynamical instability of stellar associations. Per OB 1 was considered in particular (04.153.033). Further, Mirzoyan et al. (04.152.001) derived the space distributions of O-B5 stars in the synthetic association and estimated mean ages for stars of various spectral-type intervals, assuming stationary ejection from the association nucleus. Rybakov et al. (05.152.010) maintain that observed expansions must not be taken as proof of disintegration, because the total masses are unknown.

Meurers (Vienna) investigated systematic effects in artificial two-dimensional velocity-fields, produced by random-number generators. The result is that expansion among associations may represent accidental phenomena.

The development of stellar associations was studied by Arny (03.152.008) by means of a simple model containing initial condensations. To avoid difficulties with gravitational contraction for the origin of loose associations, Pikelner (03.152.007) considered a mechanism involving heating and ionization of the interstellar gas by low-energy cosmic rays, or more probably by low-energy X-rays.

\section{STRUCTURE AND DYNAMICS OF CLUSTERS}

Many of the investigations mentioned in Section 4 are partly dealing with structural properties of clusters. Studies of stellar distributions in open clusters include papers by Artiukhina on the $\alpha$ Per cluster (07.153.015) and on M39 (03.153.004), by Artiukhina and Kholopov (04.154.016) on NGC 7209, and by Kholopov (05.153.034) on the Pleiades. NGC 2264 was studied by Götz (06.153.023) in a first paper on the structure of the very young clusters. Feinstein and Marraco (La Plata) found that the clusters $\operatorname{Tr} 14, \operatorname{Tr} 16$, and $\mathrm{Cr} 232$ in the region around $\eta$ Car all belong to a single physical group of very young characteristics. The group is embedded in dust and is probably connected with the emission nebula NGC 3372 . On the other hand, $\eta$ Car itself is situated in a small region with very little dust.

Lohmann (06.153.003) determined structural parameters for 20 open clusters from star counts. Syrovoj discussed the classification problem (04.153.034) and the distribution of mean stellar masses (05.153.041). Improved mass and luminosity functions were derived by Popova and Valts (01.153.019), and Yokoo (07.153.030) investigated the mass spectrum of the Hyades.

From star counts Lohmann (07.153.023) derived total masses for 39 open clusters and determined the mean stellar velocities. Jones studied the internal motions in the Pleiades (03.153.020) and Praesepe (05.153.037). Outlying stars in the Pleiades appear to show a striking preference for radial motion (03.153.001). 
Preliminary results from observations of neutral hydrogen in the regions of four open clusters were presented by Tovmassian (03.153.015). D'Odorico and Felli (03.153.002) studied the correlation between the mass of dust in clusters and the mass of neutral hydrogen in the cluster regions. Schwartz (06.153.026) observed 41 clusters in the hydrogen continuum and found thermal emission only from clusters which contain stars earlier than B1. The ratio of stellar mass to gas mass is ranging from 1 to 500 for the various clusters, and the ratio of gas to dust mass from 20 to 500 .

The age distribution and total life-times of open clusters were studied by Wielen (05.153.040). The great dearth of old clusters must be explained by their dissolution through dynamical effects. Evaporation, assisted by the galactic tidal field, is the major contributor; but tidal shocks are probably also important.

In addition to structural studies of globular clusters mentioned in Section 4 we note a paper on M4 by Castellani et al. (50). Kukarkin revised the concentration classes (05.154.004), studied the influence on the diameters of interstellar and atmospheric absorption (04.154.018), and investigated the correlation between diameter and metallicity (04.154.019). Mnatsakanyan (03.154.018) discussed the problem of finding the stellar distribution in poor clusters. Observational aspects of hypothetical black holes in cluster centres were considered by Wyller (03.154.015).

Freeman and Illingworth (Mt Stromlo) developed a method for determining masses for globular clusters from the luminosity profile and the central velocity-dispersion, obtained by means of image-tube spectra. For NGC 6383 the resulting mass is $3 \times 10^{8} M_{\odot}$ and the mass to visual-light ratio about 7 , with an uncertainty by a factor 2 .

Kerr and Knapp (51) observed 12 globulars in the search for 21-cm emission at the appropriate velocity. None of the clusters showed any emission. More recently Knapp et al. (52) made more sensitive observations of eight clusters, again with no detection of $\mathrm{HI}$. The limits set range from $0.8 M_{\odot}$ for $\mathrm{M} 22$ to $27 M_{\odot}$ for NGC 6293. Kerr and Knapp (53) also searched for maser emission in the $1612 \mathrm{MHz}$ OH-line from possible IR-stars in globular clusters. None of the 16 clusters observed showed any such emission down to low limits. The lack of neutral hydrogen suggests that any interstellar gas in globular clusters must be ionized. This is tentatively confirmed by Philip and Erkes (54) who report marginal detection of continuum emission at $6 \mathrm{~cm}$ from 18 globulars.

It is impossible, within the limited scope of this review, to discuss all recent theoretical studies, and only a summary of high points and general trends will be given.

A large amount of effort was devoted to numerical simulation of clusters, both by the $N$-body method and by the Monte Carlo method. $N$-body calculations are discussed at great length and in great breadth in Gravitational N-Body Problem (07.012.004). Noteworthy results include discussions of unbounded systems by Aarseth (06.042.045), tidally bounded systems by Hayli (06.151.034), tidally shocked systems by Bouvier and Janin (04.151.033), binary formation by Aarseth (06.151.035), and run-away stars by Allen and Poveda (06.151.036).

The second major simulation approach, Hénon's Monte Carlo method, is described in detail in the above volume, where Hénon (06.151.032) also includes a discussion of some results; but the most extensive applications were made by Spitzer and his co-workers $(05.151 .019 ; 05.151 .040$; 07.151.068;07.151.088).

The $N$-body and Monte Carlo methods effectively complement each other; the first follows all the effects for small $N$, such as collective effects and binary formation, while the second is better able to simulate the smoother evolution that is associated with large $N$.

On the analytical side, a numerical method of solving time-dependent problems was developed by Larson (03.151.016) and used by him for calculating the evolution of model clusters (04.151.002). In this treatment moments of the velocity distribution up to the fourth order are included.

The various methods of computation were used to study several dynamic phenomena in clusters. One is the condensation and eventual collapse of the core, which had been predicted computationally by von Hoerner and analytically by Hénon, and rationalized thermodynamically by Lynden-Bell. The clearest demonstration comes from Monte Carlo calculations by Spitzer and Thuan (07.151.088), who show that over a wide range of conditions collapse of a core takes place in 18 times the initial relaxation-time of the central half of the system. 
The formation of a dense core is furthered by the interaction between stars of high and low mass. Equipartition fails as an approximation: even when the mass function is fairly level, the low-mass stars fall short of equipartition energies, and a mass function that is steeply inclined toward high masses turns equipartition tendency into a central collapse of the massive stars (02.151.038).

A related problem is the formation of binaries, which tend to involve massive stars and to dominate the evolution of the core (06.151.035). For large $N$, however, binary formation becomes much less important (05.151.019).

Two new dynamical phenomena have been studied that may be important in the evolution of clusters. One is the binding, by conservation of a pseudo-integral quantity, of stars whose energy would otherwise allow them to escape in a tidal field $(04.042 .034 ; 04.042 .075)$. The second phenomenon is the compressive tidal shock given to a globular cluster as it passes through the galactic plane (55); this phenomenon may be as important as relaxation in determining the dynamical evolution of some clusters.

The dynamical evolution of a rich open cluster, such as M67, was studied by Prata (06.151.065; 06.151.066) taking into account tidal limitation, star loss, gas loss, and tidal shocks. Aarseth and Hills (56) discussed the evolution of a cluster with initial sub-clustering, and Aarseth and Woolf (57) investigated the depletion of low-mass stars in $N$-body calculations that include tidal effects and super-nova events. A general review on the evolution of clusters and associations was given by Bouvier (05.151.035).

\section{CLUSTERS AND STELLAR EVOLUTION}

\section{A. Very young clusters and associations}

The presence of circumstellar shells of gas and dust around many of the A-F type contracting stars in NGC 2264 was found by Strom and co-workers $(05.153 .026 ; 07.153 .003)$ from uvby and $\beta$ photometry, infra-red photometry, and from hydrogen-line profiles. The absorption is nearly grey, indicating large particle sizes. Similarly, Smith (7) found evidence for circumstellar gas emission among the (mainly A-type) stars in the Ori OB 1c association. Neutral absorption by these shells can explain a large part of the scatter of the A-F type contracting stars in the c-m diagram, but Smith finds that a dispersion in the age of the stars is still required. Additional evidence for circumstellar shells was reported by Breger (07.153.007), who found that the stars also tend to show irregular light-variations, and polarization in some cases. Breger and Dyck (07.132.035) found that the wave-length dependence of the polarization matches that observed in Be stars, and concluded that in both cases it is produced by circumstellar matter.

Earlier observations by Walker, quoted in the 1967 report, had shown that many stars in NGC 2264 and in the Orion Nebula, still fainter than those discussed above, have a strong blue continuum. It was pointed out that the effect of this is to move the star to the left in the c-m diagram, making it lie close to or even below the main sequence. The existence of this effect for the UV-excess stars was demonstrated by Walker (07.114.122). It was also shown by Andrews (03.132.032), who found a correlation between UV-excess and distance from the ZAMS for a large sample of flare stars in Orion.

Taken together, these results indicate that throughout the domain in the $\mathrm{c}-\mathrm{m}$ diagram populated by gravitationally contracting stars, the location of these stars in the $\left(M_{\mathrm{bo1}}, \log T_{\mathrm{e}}\right)$-plane can not be inferred directly from the photometry; spectroscopic observations of individual stars are needed. This is of importance in connection with recent theoretical studies attempting to account for the fact that the faint stars are closer to the main sequence than predicted for a cluster of coeval stars. Ulrich $(05.153 .027 ; 06.065 .014)$ found that the observed sequence in NGC 2264 and in the Orion Nebula cluster could be explained by assuming a higher than normal abundance of ${ }^{3} \mathrm{He}$. Bodenheimer and Ostriker (04.065.063) calculated pre-main-sequence evolutionary tracks for 3-12 $M_{\odot}$ stars, and concluded that the observed spread in luminosity for stars of the same mass and age in NGC 2264 could be due to random orientation of the axes of rotation. Kuhi and 
Forbes (03.065.016), and Ezer and Cameron (05.065.026), calculated evolutionary tracks with mass loss, and found that mass loss during contraction has little effect, and thus that the observed $c-m$ diagrams of very young clusters cannot be explained on this basis. In each of these investigations, the normal relation between $B-V$ and $T_{\mathrm{e}}$ was assumed.

Spectroscopic observations of 25 of the UV-excess stars in the Orion Nebula and NGC 2264 were published by Walker (07.114.122). In nine of the brightest stars, an inverse P Cyg spectrum is observed, indicating infall of gas. According to calculations by Larson $(02.065 .036 ; 07.065 .077)$ of the initial stages of star formation, a small part of the collapsing cloud will rapidly condense into a stellar core, which then grows by accreting the remaining material. The stellar core is completely obscured by dust in the infalling cloud, until nearly all the cloud material has been assimilated. The observed UV-excess stars occur just at that point in the $\left(M_{\mathrm{bol}}, \log T_{\mathrm{e}}\right)$-plane where stars in the relevant mass range should, according to Larson's theory, first become visible through the remnants of the pre-stellar cloud. The ages of these stars range from $3 \times 10^{4}$ to $1 \times 10^{6} \mathrm{yr}$, and are considerably smaller than the age inferred from the arrival point on the main sequence. Thus, a time-spread of star formation appears to exist, a conclusion also drawn by Strom (58) in a recent review.

The IR-sources in young clusters continue to be of great interest. Becklin's object in the Orion Nebula was discussed by Larson (02.065.037), who calculated the spectrum expected from a protostar still surrounded by the remains of the pre-stellar cloud, and by Low et al. (03.113.027), who made additional photometry, and concluded that the object appears to be a dust cloud containing a proto-star. Penston et al. (06.113.038), on the other hand, conclude that it is a highly reddened normal star, but this conclusion is disputed by Larson (07.065.077). An IR-object was found in NGC 2264 by Allen (07.114.027) and observed in the visual region by Schmidt (22). Another IR-object was found by Gillett and Stein (03.132.008) in the brightest condensation of the 'hour glass' in M8, which is associated with the young cluster NGC 6530.

Two new types of objects have been detected in young clusters. Breger (07.153.007) found two $\delta$ Sct variables in NGC 2264, indicating that this type of variability can occur in pre-main-sequence stars located within the instability strip. Smith (6) found five Am stars in the Ori OB 1c association. These are pre-main-sequence stars, located in the same region of the HR diagram as the evolving Am stars. This discovery indicates that the Am mechanism becomes operative in less than about $10^{6} \mathrm{yr}$, and suggests that stellar rotation rather than age is the dominant factor; Am stars occur more frequently in clusters where the rotational velocities are average or low. It is interesting to note that Abt et al. (04.152.007) found that in the Orion Nebula cluster, the early B stars show slow rotation, while the late $B$ stars show more rapid rotation than similar stars in the association, and the A stars show very high rotational velocities compared to field stars. Thus, differences in rotational velocity between the cluster and the association appear to exist.

The problem of star formation in clusters of small mass is of considerable interest, as discussed in the 1970 report. Aveni and Hunter (07.065.047) continued their observational studies of groups of this type, and obtained data for six early-type clusters associated with reflection nebulae.

As to the stellar associations it may be mentioned that new nuclear ages were derived by Taviev et al. (05.152.006), and by Stothers (59). Maeder (60) finds that statistical effects very often lead to large over-estimates of the nuclear ages and that there is really no systematic difference with respect to the kinematic ages.

\section{B. Open clusters of older age}

Clusters old enough to have well-populated main sequences often show considerable spread on the observed sequences, one of the many reasons being star formation during an extended timeinterval. Hartmann (05.153.023), discussing star formation and the initial mass function, derived a value as large as $2 \times 10^{8} \mathrm{yr}$ for the duration of star-formation activity in open clusters. Schlesinger (05.153.031) calculated the stellar distribution in the $\mathrm{c}-\mathrm{m}$ diagram, using two different massfunction laws, and (61) computed c-m diagrams for synthetic clusters, assuming an age-spread of $1.5 \times 10^{7} \mathrm{yr}$ for member stars. 
Another reason for increased spread is occurrence of Ap and Am stars. As pointed out by Renson (05.155.038), there is a high proportion of such stars in certain clusters. Am stars are found also in young clusters (04.153.037), and in associations, as mentioned in the previous sub-section. The short-period variability of both Am stars and non-metallic A stars in clusters was investigated by Breger $(01.153 .006 ; 04.153 .040 ; 19 ; 62)$. Significant correlations were established between slow rotation, metallicity, and pulsational stability.

For galactic early-type clusters the scarcity of stars and the spread make it difficult to determine ages, from computed isochrones, with the accuracy that might be expected (03.065.017). The situation is more favourable for the star-rich blue clusters in the Magellanic Clouds. Thus, for the LMC cluster NGC 2136 Robertson (Mt Stromlo) derived the age $1.8 \times 10^{7} \mathrm{yr}$ and the age spread $1 \times 10^{7} \mathrm{yr}$. Models of $5 M_{\odot}$ with improved envelope-opacities were used by Robertson ( 07.065 .081$)$ for a rather satisfactory representation of the c-m diagram of NGC 1866 in the LMC. Robertson's (63) computations for other mass-values confirm that most giants and super-giants, found in young clusters, are in the core-helium-burning stage. These computations, as well as previous work by Chiosi and Summa (04.065.043), and Simpson (05.065.065), emphasize the rôle of semi-convection for the theoretical interpretation. Further arguments were given by Stothers (64) for explaining the paucity of very massive red super-giants by the effect of neutrino losses, occurring during carbon burning and later evolutionary phases. Stothers (59) also made an extensive comparison between fundamental data for massive stars and the theoretical predictions.

Gaps on the evolved main-sequences of old open clusters have now been found in several other cases than M67 and NGC 188. Demarque and Miller (02.153.028) discussed the gaps of NGC 2360 and 3680, while Cannon and Lloyd (02.153.005) studied NGC 6939. Hawarden (05.153.044) found the properties of the gaps to be related to the properties of giant-branch clumps. Cannon (04.153.003) made a detailed comparison between the theoretical predictions and observations of red giants in old clusters. Demarque and Heasley (05.153.005) suggested that both helium content $Y$, and metal content $Z$, could be determined from the properties of gaps. In another paper, Demarque and Heasley (06.065.073) computed the red-giant evolution of a $1.19 M_{\odot}$ super-metal-rich star and compared with the clumpings on the giant branch.

Using new photometric data, Sandage and Eggen (02.153.023) discussed in detail the ages and evolutionary stages of M67 and NGC 188. Ages were determined from two sets of isochrones computed from models due to Aizenman, Demarque, and Miller $(X=0 \cdot 67, Z=0.03)$, and to Iben $(X=0.708, Z=0.020)$. For M67 the age was found as $5.5 \times 10^{9} \mathrm{yr}$, while for NGC 188 the two sets of isochrones gave ages in the interval $(8-10) \times 10^{9} \mathrm{yr}$. A new version of the composite $c-m$ diagram for clusters of old and intermediate age is presented in the paper. We note here that van den Heuvel (02.153.008) derived revised ages, about $9 \times 10^{8}$ yr for Praesepe and the Hyades, and about $6.5 \times 10^{8} \mathrm{yr}$ for the Coma cluster.

Following Spinrad and Taylor's (02.114.115; 05.153.004) conclusion that M67, NGC 188, and NGC 6791 are super-metal-rich, Torres-Peimbert (06.065.128) computed stellar models with high metal-content. From the isochrones Torres-Peimbert (06.153.002) found the best fit with $Z=0.06$ for NGC 188 and with $Z=0.10$ for NGC 6791, while for M67 the isochrones failed to give any information on $Z$, and $Z=0.06$ was assumed in conformity with Spinrad and Taylor. The resulting ages are about half those derived by Sandage and Eggen. Demarque and Gisler (65) have, however, recently computed models with improved opacities and for a range of $Z$, and they find in a preliminary analysis that super-metallicity seems unnecessary for the interpretation of the c-m diagram of NGC 188. Similarly, Hejlesen et al. (66) find a normal metal content $(Z \approx 0.02, X \approx 0.80)$ for M67, when comparing with their grid of models computed with the Cox-Stewart opacities. The corresponding age is about $3 \times 10^{9} \mathrm{yr}$.

Several of the blue stragglers in NGC 7789 were shown by Strom and Strom (04.153.039) to be cluster members and to exhibit radial-velocity variations, indicating that they are members of binary systems in which mass exchange has occurred. Studies by Bond and Perry (06.153.022), and by Strom et al. (06.153.029), indicate that the binary explanation is also true for some stars forming a pseudo-horizontal branch in the c-m diagram of M67. Eggen (06.122.158) found, 
however, more evidence for short-period variables than for binaries among the blue stragglers in old clusters and stellar groups, and he argues that the question should be re-examined.

\section{Globular clusters}

From photometry down to faint limits, $V \approx 22 \cdot 0$, Sandage (04.154.017) fitted together the c-m diagram of M3, M13, M15, and M92, using field sub-dwarfs with known parallaxes for calibration and taking the UV excesses into account. The main-sequence turn-off is for M3 and M13 found at $M_{V} \approx 4 \cdot 10$, and for M15 and M92 at $M_{V}=3 \cdot 80$. These values were reduced to $M_{\mathrm{bo} 1}$, taking blanketing effects into consideration. Ages were determined from models computed by Iben and Rood (03.065.012) and by Demarque et al. (05.065.005), using interpolation formulae with the atmospheric helium content $Y$, the metal content $Z$, and the turn-off luminosity as parameters. Sandage determined $Y$ from the pulsation theory and $Z$ from spectroscopic data. The obtained values of $Y$ are slightly larger than $\mathbf{0 . 3 0}$ for all the clusters, while $Z$ ranges from 0.00015 for M92 and M15 to 0.0008 for M13. These values inserted in the interpolation formulae give ages close to $10 \times 10^{9} \mathrm{yr}$ for all four clusters.

Comparisons between observed and theoretical luminosity functions are a powerful means for checking theoretical assumptions and predictions. The problems involved were reviewed by Castellani and D'Antona (06.154.018), and by Iben (06.154.021). Hartwick (04.154.009) determined the empirical luminosity function for M92, Simoda and Tanikawa (07.154.002) those for M5 and M13, while Simoda and Fukuoka are working on M3. When comparing with theory, Hartwick (04.154.018) found $Y \approx 0.35$ for M92, and about the same value was derived by Faulkner (07.154.019) in a re-discussion, using theoretical luminosity functions due to Simoda and Iben (04.154.008). Simoda (07.154.003) discussed M5 and M13, as well as Hartwick's data for M92, and found from the sub-giant region high $\mathrm{He}$ content for all three clusters. Comparisons of the horizontal-branch (HB) luminosity functions, with the life-times expected from Iben and Rood's (04.065.023) corehelium and hydrogen-shell-burning models, again indicate high $\mathrm{He}$ abundance.

The giant sequences of several clusters exhibit gaps and clumps, which in many cases might be statistically significant $(07.154 .003 ; 07.154 .015)$. Demarque et al. $(07.154 .013)$ conjectured that certain gaps may be explained by fast rotating cores in the red giants, the core boundaries being traversed by the outward-moving hydrogen-burning shells.

The physical characteristics of the red giants, thought to have masses about $0.8 M_{\odot}$, are studied photometrically by Osborn $(07.154 .030 ; 67)$, using a modification of the DDO six-colour system. The photometry allows determination of parameters, such as $Z, T_{\mathrm{e}}$, and $\log g$, for typical cluster stars.

After the helium flash, terminating the first red-giant-stage, the stars move rapidly to the horizontal branch, if no mixing occurs. The locus of the initial HB models (04.065.008; 07.065.024), the mode of evolution, and the HB life-time (04.065.023) are highly dependent upon total mass, core mass, and envelope He content. Assuming plausible mass values, the number ratio of HB stars to red giants will provide an expedient way to gain information on $Y$. This method, used by Iben and collaborators $(02.154 .018 ; 02.154 .019 ; 06.154 .021)$, has given $Y \approx 0.30$. Demarque et al. (68) point out that semi-convection, if important for HB stars, will prolong the life-times, and the observed number ratios will indicate much lower He abundances. Although not strictly demonstrated, it is probable that semi-convection is of importance for these He-burning stars (69). On the other hand, values of $Y \approx 0.30$ seem so firmly established by various methods that Demarque et al. (68) look for other escapes from the dilemma.

The physical data of HB stars were discussed by van Albada and Baker (06.154.013), and by Thänert (06.065.023), using relations derived for the RR Lyr variables. Stobie (06.154.010), and van Albada and Baker (07.154.033), studied the differences between the Oosterhoff types I and II clusters, exemplified by M3 and $\omega$ Cen, respectively. In a comprehensive review, Iben (06.154.021) compared results from theoretical studies of evolution and pulsation with the observations. As shown by Iben, it should be possible to determine both $\mathrm{He}$ abundance and luminosity of the RR Lyr stars from the positions of the blue edge of the instability strip and the transition edge 
between $a b$ and $c$ variables. The problem was further discussed by Iben and Tuggle (07.122.025), and an application to several clusters was attempted.

Recent investigations on the blue horizontal-branch (BHB) stars include studies mainly based on $U B V$ photometry by Newell $(03.154 .006 ; 04.154 .003)$, and $u v b y$ and $\beta$ photometry, quoted in Table 3 , by Philip and by Tifft. Summaries were given by Newell $(07.154 .032)$ and by Philip (07.154.029). In agreement with Newell, Philip (07.154.007) finds that BHB stars in metal-poor and relatively metal-rich clusters show similar ( $\log g, \theta_{\mathrm{e}}$ )-relations, but that M3 and M13, with intermediate metal-abundance, have BHB stars with lower $g$-values. Newell (07.154.032) estimates that BHB stars with $\log T_{\mathrm{c}} \leqslant 4.1$ have the average mass $0.55 \pm 0.2 M_{\odot}$. Mass loss of various amount seems necessary for explaining the observations and for populating the entire HB. Faulkner (07.065.072) pointed out that the extremely blue sequence of halo sub-dwarfs, observed by Greenstein (05.126.020), shows that substantial mass loss may occur, and that these blue stars are probably of similar nature as the BHB stars. Caloi (70) computed models for stars on the extended $\mathrm{HB}$, while Demarque and Mengel (07.065.020) commented upon the rôle of semi-convection for obtaining blue extensions of the HB.

The evolved HB stars are moving to positions above the horizontal branch (AHB stars) and to the right in the HR diagram, forming an asymptotic branch $(\mathrm{AB})$ leading to a second giant stage. When ascending the giant branch the $\mathrm{AB}$ stars are burning hydrogen and helium in shells. It was shown by Schwarzschild and Härm (03.154.012) that the inner shell passes through a series of thermal instabilities, which will produce loops in the HR diagram. Stars of low mass $\left(M=0.65 M_{\odot}\right)$ and high He content have loops that cross the cepheid instability strip. Wallerstein (03.154.013) found the observational data on population II cepheids in globular clusters to confirm this interpretation, only clusters having a well-populated BHB contain cepheids.

Stars with unusual strong $\mathrm{CN}$ and $\mathrm{CH}$ are found $(06.154 .003 ; 06.154 .016 ; 40)$ among the red $\mathrm{AB}$ stars. They may represent mixed stars, with atmospheric $\mathrm{H}$ depleted. Various problems concerning AB stars were reviewed by Strom and Strom (05.154.010), and by Castellani and D'Antona (07.115.006).

The final evolution will carry the stars off the asymptotic branch towards higher $T_{\mathrm{c}}$ and to the white-dwarf stage (03.065.018; 07.065.070). Strom et al. (04.065.018) have in the AHB region of some clusters found a few luminous stars, which probably are in the post-AB evolution phase. A large number of UV-bright stars were located by Zinn et al. (07.154.017) in the AHB regions of 27 clusters. Hopefully, some of these objects, to be studied by other means, might be cluster members and post-AB stars.

Much additional material on the evolution of globular-cluster stars is found in the proceedings of the Stony Brook conference (07.012.024). Attention is also drawn to Schwarzschild's (03.154.005) George Darwin lecture.

Among other studies partly dealing with evolutionary problems, we note a paper by Bell (03.154.017) on the interpretation of the $\mathrm{c}-\mathrm{m}$ diagram of M92, an investigation of the stellar content of halo-type clusters by Talbert (03.154.021), a general study of the HR diagrams by Castellani et al. (01.154.005), and a multimensional cluster classification by the same authors (04.154.012). D'Antona (06.065.116) confirmed the validity of the $Q$ index as a metallicity parameter, while Young (04.154.005) and Mironov (07.154.005) critizised Hartwick's two-dimensional classification. Hartwick and McClure (71) now find from a study in the DDO system of the anomalous cluster NGC 7006 that the nitrogen abundance, rather than that of helium, might possibly be the second parameter, which, together with metallicity, is correlated to the HB structure.

\section{CLUSTERS AND GALACTIC STRUCTURE}

Work using clusters as a tool for the study of galactic structure is considered to fall generally outside the domain of Commission 37. Only some few investigations, where the clusters as such are of major importance, will be mentioned here.

The distance of the Hyades was discussed by several authors, including Eggen (01.153.018; 
02.153.029), Upton (04.153.038), and Golay (72). The method used by Upton is based on a new treatment of stellar motions, and involved also Praesepe. The distances obtained for the two clusters are in good agreement with recent analyses by the convergent-point method. In another related paper, Upton (05.153.013) calibrated the zero-age main sequence by fitting the Praesepe curve to G8-K3 stars within $13 \mathrm{pc}$ from the Sun. A recalibration of the absolute magnitudes of supergiants was performed by Stothers (73) using data from clusters and associations.

Eggen's (01.153.018) result that the mass-luminosity relation of the Hyades group is different from that of the Sun-Sirius group, implying higher He content for the Hyades-group stars, was re-examined by Alexander (07.115.011). He finds that unevolved stars in the Hyades are probably somewhat undermassive, when compared to typical unevolved field stars of corresponding luminosity, but he cannot find evidence for two discrete mass-luminosity relations among field stars belonging to the two groups.

Absolute proper motions on the FK4 system were derived for 61 open clusters by von Schewick (06.153.020). Measurements by the author and reduced data from other sources were used separately or in combination. The solar motion and values of Oort's constant $B$ were determined. Determinations of galactic kinematical parameters from the radial velocities of clusters were made by Buscombe (74), and by Barkhatova and Danilov (Ural University). Syrovoj (05.155.047) discussed galactic orbits of open clusters. Isobe (07.155.004) calculated space velocities of 12 open clusters and commented upon the large $z$-motions previously found for some young clusters.

\section{SUMMARY AND OUTLOOK}

The study of star clusters and associations has during the past triennium been pursued vigorously by a large number of astronomers. Observers in the southern hemisphere are starting to furnish data for the many southern clusters that have remained almost unobserved until now. Especially promising for future work are the star-rich clusters in the Magellanic Clouds. Some of these are now being studied with the electronographic technique.

In the past cluster members have generally been segregated by means of photometric data, perhaps sustained by spectral types. For the very many faint clusters this will remain the only feasible method. It means, however, that cluster members, which are photometrically peculiar, as a rule will be dismissed. Membership studies using proper motions are now gaining importance, as for several clusters the time intervals to first-epoch plates have grown large enough. Equally useful are radial velocities, and the development of the fast and accurate photoelectric technique for their measurement holds great promise for the future. Already it has produced remarkable results in cluster work.

Clusters and associations are important in many respects. They provide information on star formation and stellar evolution, and they constitute the ideal objects for testing theories in this field. It has recently been possible to trace the propagation of star formation within an association. Observations of very young clusters have shown that several newly formed stars are surrounded by infalling shells of gas and dust, as theoretically predicted. Through the combined effort of observation and theory the late phases of low-mass stellar evolution are being unveiled, and observers have succeeded in locating globular-cluster stars, which may be evolving towards the final white-dwarf-stage.

The ages of the globular clusters have now been set to about $10 \times 10^{9} \mathrm{yr}$. This is satisfactory, from the cosmological point of view, as the ages of the oldest stars in our Galaxy turn out considerably lower than the Hubble age of the universe, according to the revised value of the Hubble constant. Of great cosmologic importance is also the high and uniform helium content, that seems firmly established for the globulars. The metal abundance of old population I clusters has in recent years been the subject of a heated debate, but it now appears that the metallicities are not as high as some investigators once believed.

The dynamical evolution of clusters of various size are highly interesting and intriguing. The disintegration of clusters by evaporation and tidal forces is coupled to binary formation and stellarevolution effects, such as super-nova events. Much progress has been made in this field, but much remains to be done. 
Conventional studies of clusters and associations involve determinations of distances and reddenings, quantities of direct interest to galactic research. Studies of objects affected by non-uniform absorption indicate that the ratio of total absorption to reddening is more universal than was believed some years ago. Young clusters and associations remain one of the best optical indicators of galactic spiral arms. Many fundamental calibration-problems in stellar astronomy are most satisfactorily solved by means of clusters and associations.

G. LARSSON-LEANDER

President of the Commission

\section{REFERENCES}

1. Garmany, C. D. 1972, Bull. Am. Astron. Soc., 4, 316.

2. Knacke, R. F., Strom, S. E., Strom, K. M., Young, E. $\quad$ 1972, Bull. Am. Astron. Soc., 4, 325.

3. Penston, M. V. 1972, preprint.

4. Vitrichenko, E. A. 1972, Astron. Tsirk., No. 677, 5.

5. Bernacca, P. L., Ciatti, F. 1972, Astron. Astrophys., 19, 482.

6. Smith, M. A. 1972, Astrophys. J., 175, 765.

7. Smith, M. A. 1972, Astrophys. J., 176, 617.

8. Drilling, J. S. 1972, Astron. J., 77, 463.

9. Laval, A. 1972, Astron. Astrophys., 21, 271.

10. Webster, B. L. 1972, Observatory, 92, 143.

11. Van Altena, W. F., Jones, B. F. $\quad$ 1972, Astron. Astrophys., 20, 425.

12. Sanders, W. L. $\quad$ 1973, Astron. Astrophys. Suppl., 9, 213.

13. Moffat, A. F. J. 1972, Astron. Astrophys. Suppl., 7, 355.

14. Sanders, W. L. $\quad$ 1973, Astron. Astrophys. Suppl., 9, 221.

15. Sanders, W. L. 1970, Thesis (Göttingen Univ.).

16. Percy, J. R. 1972, Publ. Astron. Soc. Pacific, 84, 420.

17. Garrison, R. F. $\quad$ 1972, J. Roy. Astron. Soc. Can., 66, 216.

18. Ianna, P. A. 1968, Thesis (Ohio State Univ.).

19. Breger, M. 1972, Astrophys. J., 176, 367.

20. Hassan, S. M. 1972, Mem. Soc. Astron. Ital., 43, 279.

21. Burkhead, M. S., Burgess, R. D., Haisch, B. M. 1972, Astron. J., 77, 661.

22. Schmidt, E. G. $\quad 1972$, Astrophys. J. Letters, 176, L69.

23. Fenkart, R. P., Buser, R., Ritter, H., Schmitt, H., Steppe, H., Wagner, R., Wiedemann, D. 1972, Astron. Astrophys. Suppl., 7, 487.

24. Vogt, N., Moffat, A. F. J. $\quad 1972$, Astron. Astrophys. Suppl., 7, 133.

25. Vogt, N., Moffat, A. F. J. 1973 , Astron. Astrophys. Suppl., 9, 97.

26. Tifft, W. G., Connolly, L. P., Webb, D. F. 1972, Monthly Notices Roy. Astron. Soc., 158, 47.

27. Lindoff, U. 1972, Astron. Astrophys. Suppl., 7, 231.

28. Glaspey, J. W. 1972 , Astron. J., 77, 474.

29. Eggen, O. J. 1972, Publ. Astron. Soc. Pacific, 84, 406.

30. Strom, K. M., Strom, S. E., Knacke, R. F., Young, E., Kunkel, W. $\quad$ 1972, Bull. Am. Astron. Soc., 4,325 .

31. Bond, H. E. $\quad 1972$, Publ. Astron. Soc. Pacific, 84, 583.

32. Strom, K. M., Strom, S. E., Breger, M., Brooke, A. L., Yost, J., Grasdalen, G., Carrasco, L. $\quad 1972$, Astrophys. J. Letters, 176, L93.

33. Fehrenbach, C., Duflot, M. 1972, Observatory, 92, 145.

34. Bürki, G. 1972, Travail de diplôme (Genève Univ.).

35. Bürki, G., Maeder, A. 1973 , Astron. Astrophys., in press.

36. Maeder, A. 1971, Thesis (Genève Univ.).

37. Gottlicb, D. M., Bell, R. A. $\quad 1972$, Astron. Astrophys., 19, 434.

38. Barry, D. C., Cromwell, R. H. 1972, Bull. Am. Astron. Soc., 4, 326.

39. Philip, A. G. D. 1972, Bull. Am. Astron. Soc., 4, 325.

40. Dickens, R. J. 1972, Monthly Notices Roy. Astron. Soc., 159, 7P.

41. Dickens, R. J., Feast, M. W., Evans, L. T. 1972, Monthly Notices Roy. Astron. Soc., 159, 337.

42. Stock, J., Wing, R. F. 1972 , Bull. Am. Astron. Soc., 4, 324.

43. Dickens, R. J., Rolland, A. $\quad 1972$, Monthly Notices Roy. Astron. Soc., 160, 37. 
44. Dickens, R. J., Flinn, R. 1972, Monthly Notices Roy. Astron. Soc., 158, 99.

45. Hartwick, F. D. A., McClure, R. D. 1972, Astrophys. J. Letters, 176, L57.

46. Walker, M. F. $\quad$ 1972, Monthly Notices Roy. Astron. Soc, 159, 379.

47. Feast, M. W. $\quad$ 1972, Monthly Notices Roy. Astron. Soc., 159, 113.

48. Ford, H. C. 1970, Thesis (Univ. of Wisconsin).

49. Andrews, P. J., Evans, T. L. $\quad$ 1972, Monthly Notices Roy. Astron. Soc., 159, 445.

50. Castellani, V., D'Antona, F. A., Natta, A. 1972, Astrophys. Space Sci., 17, 23.

51. Kerr, F. J., Knapp, G. R. 1972, Astron. J., 77, 573.

52. Knapp, G. R., Kerr, F. J., Rose, W. K. 1973, Astrophys. J. (paper to be submitted).

53. Kerr, F. J., Knapp, G. R. 1973, Astron. J. (paper to be submitted).

54. Philip, A. C. D., Erkes, J. W. 1972, Bull. Am. Astron. Soc., 4, 399.

55. Ostriker, J. P., Spitzer, L., Chevalier, R. A. 1972 , Astrophys. J. Letters, 176, L51.

56. Aarseth, S. J., Hills, J. G. 1972, Astron. Astrophys., 21, 255.

57. Aarseth, S. J., Woolf, N. J. $\quad$ 1972, Astrophys. Letters, 12, 159.

58. Strom, S. E. $\quad$ 1972, Bull. Am. Astron. Soc., 4, 324.

59. Stothers, R. 1972, Astrophys. J., 175, 431.

60. Maeder, A. 1972, preprint, IAU Colloquium No. 17.

61. Schlesinger, B. M. 1972, Astron. J., 77, 584.

62. Breger, M. 1972, Astrophys. J., 176, 373.

63. Robertson, J. W. 1972, Astrophys. J., 177, 473.

64. Stothers, R. 1972, Astrophys. J., 175, 717.

65. Demarque, P., Gisler, G. 1972, Bull. Am. Astron. Soc., 4, 326.

66. Hejlesen, P. M., Jørgensen, H. E., Petersen, J. O., Rømcke, L. $\quad$ 1972, preprint.

67. Osborn, W. H. 1971, Thesis (Yale Univ.).

68. Demarque, P., Sweigart, A. V., Gross, P. G. $\quad$ 1972, Nature Phys. Sci., 239, 85.

69. Sweigart, A. V., Demarque, P. 1972, Astron. Astrophys., 20, 445.

70. Caloi, V. 1972, Astron. Astrophys., 20, 357.

71. Hartwick, F. D. A., McClure, R. D. 1972, Astrophys. J. Letters, 176, L57.

72. Golay, M. 1972, preprint, IAU Symposium No. 54.

73. Stothers, R. $\quad 1972$, Publ. Astron. Soc. Pacific, 84, 373.

74. Buscombe, W. 1972, Observatory, 92, 141. 